\title{
Presentation of a highly tuned multithreaded interval solver for underdetermined and well-determined nonlinear systems
}

Empirical evaluation of innovations

\author{
Bartłomiej Jacek Kubica
}

Received: 1 September 2014 / Accepted: 16 February 2015 / Published online: 24 March 2015 (C) The Author(s) 2015. This article is published with open access at Springerlink.com

\begin{abstract}
The paper summarizes author's investigations in tuning a multithreaded interval branch-and-prune algorithm for nonlinear systems and presents the developed solver. New results for using the box-consistency enforcing operator and a new variant of the initial exclusion phase are presented. Also, a new heuristic to choose the coordinate for bisection is considered. Extensive numerical experiments are analyzed to provide the satisfying version of the algorithm.
\end{abstract}

Keywords Interval methods · Nonlinear systems of equations · Heuristics · Low-discrepancy sequences · Multithreaded computations

\section{Introduction}

We consider the problem of finding all solutions of nonlinear systems of equations, i.e., systems of the form:

$$
\begin{aligned}
& f(x)=0, \\
& x \in[\underline{x}, \bar{x}],
\end{aligned}
$$

where $f: \mathbb{R}^{n} \rightarrow \mathbb{R}^{m}, m \leq n$.

Such systems are ubiquitous in several branches of science and engineering. Many of them are not well-determined, but underdetermined, i.e., having fewer equations than unknowns $(m<n)$, which means they have uncountably many solutions and their solution sets do not consist of isolated points, but are manifolds. In particular,

B. J. Kubica (ه)

Institute of Control and Computation Engineering, Warsaw University of Technology,

Nowowiejska 15/19, 00-665 Warsaw, Poland

e-mail: bkubica@elka.pw.edu.pl 
we encounter such systems in robotics [19], stability theory of dynamical systems [35], differential equations solving [31] and multicriteria analysis [30].

Example As a specific example, we can consider solving the inverse kinematic problem of a serial planar $n R$-manipulator, i.e., a manipulator working in the $X O Y$ space and consisting of $n$ rotational joints. Assume, the kinematic chain starts in the point $(0,0)$ and the effector is supposed to be placed in the point $(1,1)$ and oriented orthogonally (under the right angle) to the $O Y$ axis. This problem can be formulated as the following system of equations:

$$
\begin{aligned}
& \sum_{i=1}^{n} l_{i} \cdot \prod_{j=1}^{i} \cos \left(\sum_{k=1}^{j} x_{k}\right)-1=0, \\
& \sum_{i=1}^{n} l_{i} \cdot \prod_{j=1}^{i} \sin \left(\sum_{k=1}^{j} x_{k}\right)-1=0, \\
& \sum_{i=1}^{n} x_{i}-\frac{\pi}{2}=0, \\
& x_{i} \in\left[-\frac{\pi}{2}, \frac{\pi}{2}\right], \quad i=1, \ldots, n .
\end{aligned}
$$

We assume $l_{i}=1.0$ for $i=1, \ldots, n$.

For $n=3$ the problem is well determined - there are exactly two manipulator configurations satisfying the constraints (see Fig. 1, on the left). But for $n=5$, the set of possible manipulator configurations is a manifold - it is of the measure continuum. A few example configurations are presented on the right part of Fig. 1

Interval methods (see, e.g., [18, 20, 37]) are a well-known approach to find all solutions of both kinds of systems. Their essence is to perform operations on (possibly multidimensional) intervals (so-called boxes in $\mathbb{R}^{n}$; see Fig. 2) instead of specific numbers (vectors), so that, if $a \in \mathbf{a}$ and $b \in \mathbf{b}$, then $(a \odot b \in \mathbf{a} \odot \mathbf{b})$, i.e., the result of an operation on numbers belongs to the result of operation on intervals, containing the arguments. This leads to interval arithmetic operations and definitions of basic functions operating on intervals. We shall not define basic interval operations here; the interested reader is referred to several papers and textbooks, e.g., [18, 20, 37].
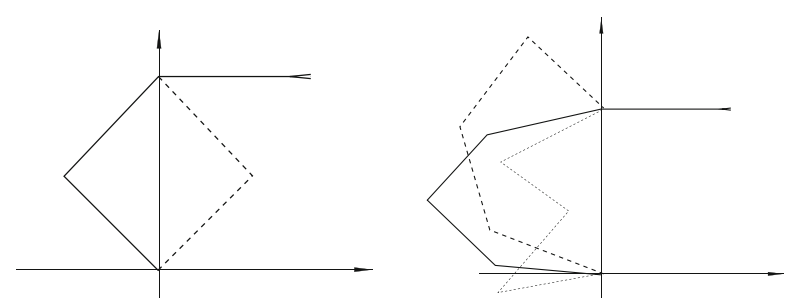

Fig. 1 Left: both feasible 3R manipulator configurations, right: three examples of uncountably many feasible $5 \mathrm{R}$ manipulator configurations 
In the previous series of papers ([22-29]) the author presented an interval solver for such systems and investigated several acceleration tools. The solver is targeted at underdetermined problems, yet it could be used for well-determined ones, also.

\section{Generic algorithm}

The solver is based on the branch-and-prune $(\mathrm{B} \& \mathrm{P})$ schema that can be expressed by pseudocode presented in Algorithm 1.

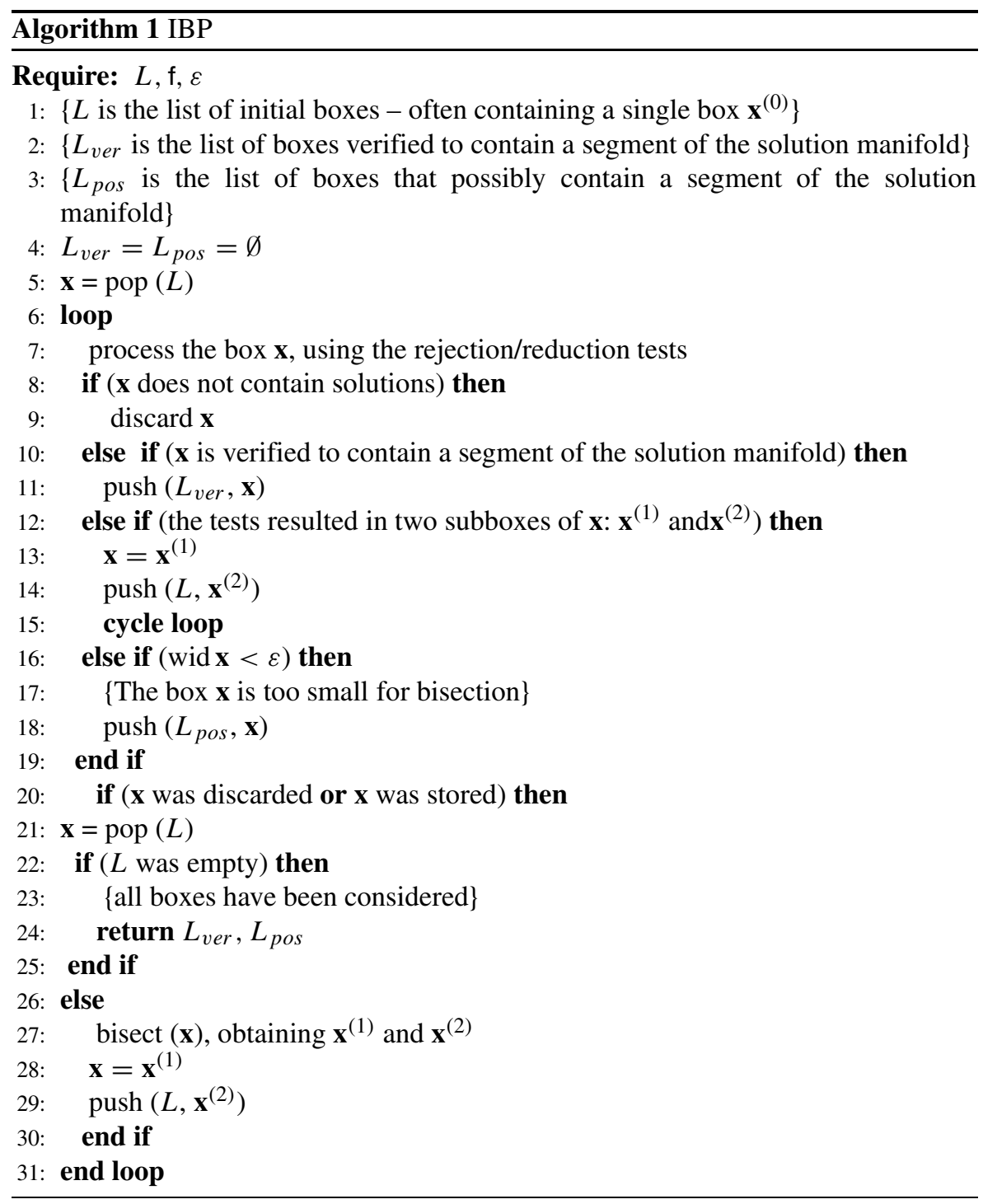



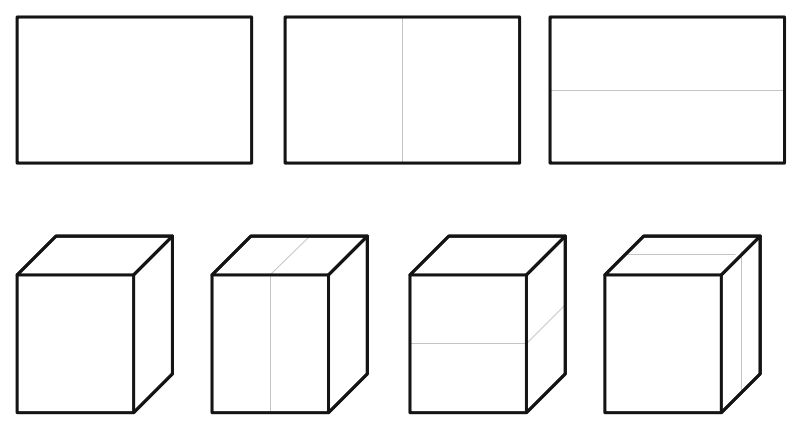

Fig. 2 Bisection of an interval and two- and three-dimensional boxes

Operations "push" and "pop" in the algorithm, mean inserting and removing elements to/from the set (the names will be used independently on how the set is represented - as a stack, queue or a more sophisticated data structure).

The precision parameter $\varepsilon$ can have various values. Usually, $10^{-7}-10^{-6}$ are sufficient values, but for hard problems (especially underdetermined ones), we have to content ourselves with larger thresholds; or the computation will take too much time.

The bisection operation ( or - to be more general - subdivision of a box) slices a box into subboxes. Usually, one of the edges of the box is splitted in the midpoint and that is the approach we use (see Fig. 2).

Algorithm 1 allows to find all solutions of the problem, yet it can be timeconsuming and memory-demanding. Because of this, it is very important to choose proper "rejection/reduction tests" (mentioned in Algorithm 1) to tune the efficiency as much as possible. Fortunately, the algorithm can be parallelized (see, e.g., [24]), as processing different boxes can be performed independently. Obviously, the lists $L$, $L_{v e r}$ and $L_{\text {pos }}$ have to be implemented in a multithreaded-safe way; so do other used tools.

The "rejection/reduction tests", mentioned above may vary. Several of them are described in previous papers of the author, specifically [27-29], i.e.:

- various kinds of the interval Newton operator and switching between the componentwise Newton operator (for larger boxes) and Gauss-Seidel with inverse-midpoint preconditioner, for smaller ones,

- a sophisticated heuristic to choose the bisected component [27],

- an initial exclusion phase of the algorithm (deleting some regions, not containing solutions) - based on Sobol sequences [28],

- an additional test based on quadratic approximation of a single equation and the Hansen's method [18] to solve quadratic equations with interval coefficients; see [29]. 
There are many other tools, also. Some of them are not suitable for multithreaded computations as they use, e.g., linear programming while popular linear programming solvers are either inefficient (e.g., the solver used in the C-XSC library [1]) or not MT-safe, e.g., the solver GLPK [5]. Hence, we do not consider some popular tools, like LP-preconditioners of [20] or LP-narrowing.

As Algorithm 1 is, in general, time-consuming and memory-demanding, it is crucial to provide a proper heuristic to choose and parameterize the rejection/reduction tests efficient for a specific class of problems.

In mentioned papers, the author considered several tools and proposed some policies to apply them. Yet, as there are so many of these tools, specific cooperation between them and tuning of the heuristics, remains to be determined.

\section{Box consistency enforcing}

One of the tools to improve the performance of Algorithm 1 are so-called consistency operators. They have not been considered in previous papers of the author. As reported, e.g., in [18], enforcing some partial consistencies can be very efficient on large boxes.

There are several kinds of partial consistencies, the most commonly used being box-consistency (BC), described, e.g., by [14] and hull-consistency (HC) - see, e.g., [10]. The latter requires complicated decomposition of the expression into a syntactic tree, so we decided not to use it in the current version of our method (unless we consider the quadratic approximation of [29] a very specific instance of HC). Hence, box consistency can be enforced using the unidimensional Newton operator that is easy to implement.

The idea of box consistency is to find the leftmost and rightmost "pseudosolutions" of a constraint [12], i.e., intervals $\left[x_{i}^{*}, x_{i}^{*+}\right]$, such that:

$$
0 \in \mathrm{f}\left(\mathbf{x}_{1}, \mathbf{x}_{2}, \ldots, \mathbf{x}_{i-1},\left[x_{i}^{*}, x_{i}^{*+}\right], \mathbf{x}_{i+1}, \ldots, \mathbf{x}_{n}\right) \text {, }
$$

where the interval $x_{i}^{*+}$ is the next representable floating-point number, after $x_{i}^{*}$, i.e., $\left[x_{i}^{*}, x_{i}^{*+}\right]$ is the smallest representable interval (such intervals are called canonical intervals).

Formula (2) is valid for equations, but it can be adapted for inequalities and other types of constraints, also.

The algorithm, we use is usually called BC3; as an analog of AC3 (AC stands for "arc consistency"; see, e.g., [11]). Usually, the algorithm is formulated as subsequent calls to recursive procedures "left_narrow" and "right_narrow", computing leftmost and rightmost pseudo-solutions. As specific implementations vary in several details (see, e.g., $[10,12,14,15])$, the author presents pseudocodes for his own implementation. Procedure "left_narrow" is described by Algorithm 2 ("right_narrow" is analogous) and the overall BC3 procedure - by Algorithm 3.

Please note, we break the procedure if the progress is not sufficient. The parameter $\varepsilon_{\text {equal }}$ is used for this purpose and the proper condition is checked in line 10 of Algorithm 3. 


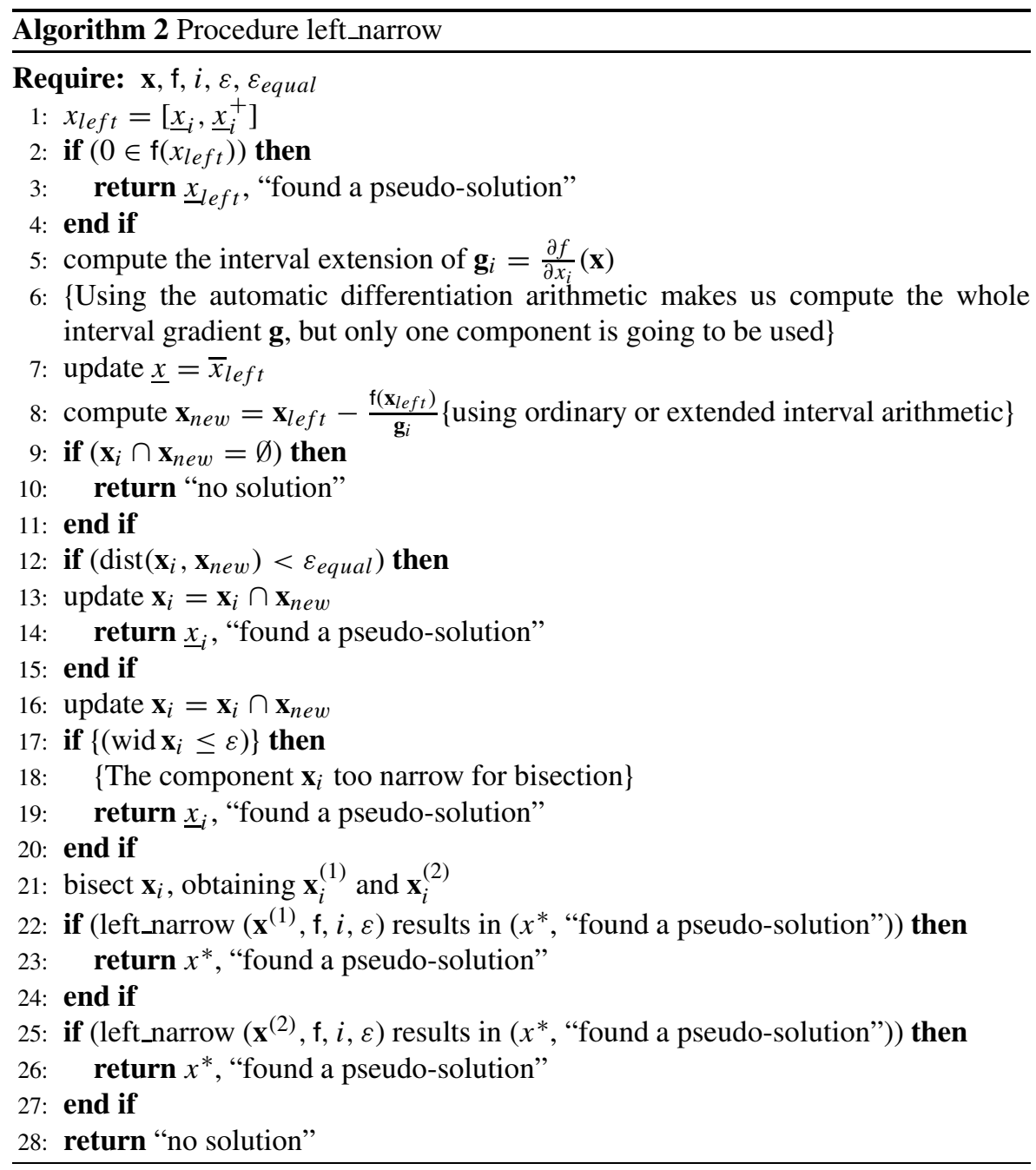

The parameter $\varepsilon_{\text {equal }}$ is used as the threshold value for braking the $\mathrm{BC} 3$ procedure - we set $\varepsilon_{\text {equal }}=10^{-4}$. Such policy is not used in other known versions of the BC3 procedure; yet, it performs well in our algorithm and seems to improve the efficiency (results proving it are not presented due to the lack of space).

Heuristic But for which boxes should we apply the BC3 procedure? For "sufficiently large", as pointed above. The heuristic we propose is described by Algorithm 4. It suffices that a single edge of the box is longer than the threshold value $\varepsilon_{b c 3}$. In Section 6 we consider two possible values of $\varepsilon_{b c 3}: \frac{3}{n}$ and $\frac{6}{n}$; results for $\frac{1.5}{n}$ are not presented as they occurred to be less promising. As for $\varepsilon_{N c m p}$, the value $\frac{1}{n}$ was used - twice larger than in [27] to emphasize the importance of the componentwise operator, partially replaced by the $\mathrm{BC} 3$ procedure. 


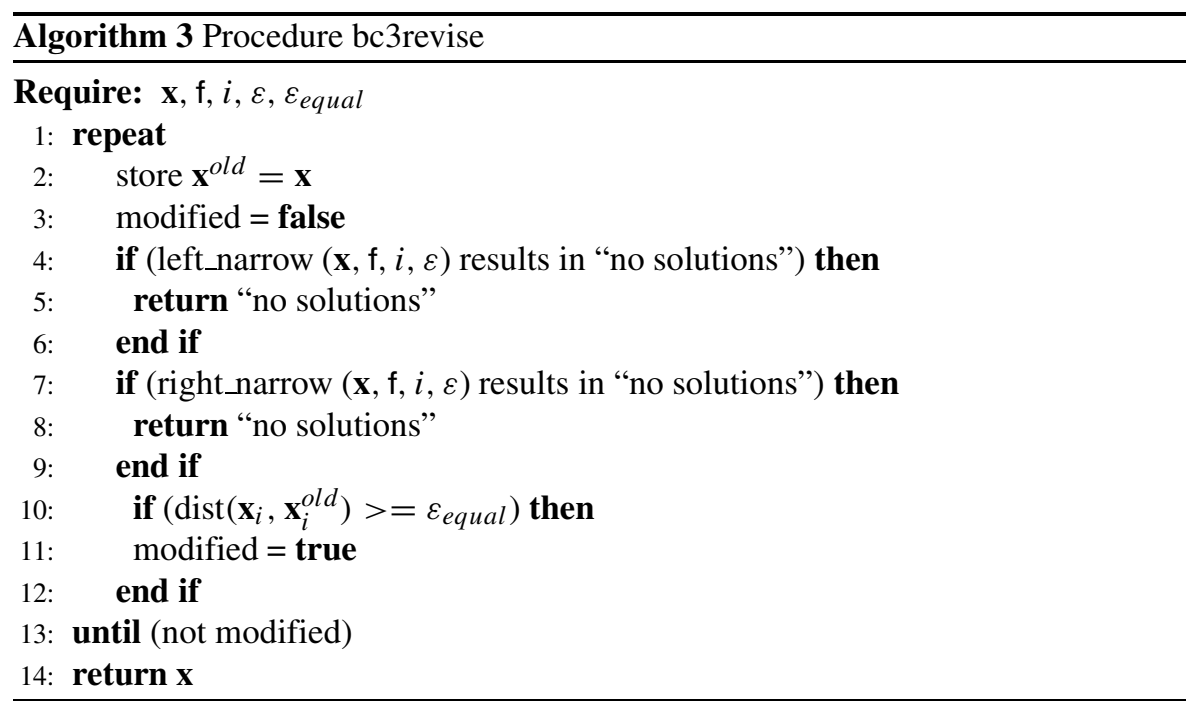

Operators Ncmp and GS are described, e.g., in [23, 25, 27]. For the sake of brevity, formulae are as follows:

$$
\begin{aligned}
& N_{c m p}(\mathbf{x}, \check{x}, \mathrm{f}, i, j)=\check{x}_{j}-\frac{\mathrm{f}_{i}\left(\mathbf{x}_{1}, \ldots, \mathbf{x}_{j-1}, \check{x}_{j}, \mathbf{x}_{j+1}, \ldots, \mathbf{x}_{n}\right)}{\frac{\partial \mathrm{f}_{i}}{\partial x_{j}}\left(\mathbf{x}_{1}, \ldots, \mathbf{x}_{n}\right)}, \\
& G S(\mathbf{x}, \check{x}, \mathrm{f}, i)=\check{x}_{i}-\left(Y_{i:} \cdot \mathrm{f}\left(\check{x}_{1}, \ldots, \check{x}_{m}, \mathbf{x}_{m+1}, \ldots, \mathbf{x}_{n}\right)+\right. \\
& \left.+\sum_{j=1, j \neq i}^{m} Y_{i:} \cdot \mathbf{J}_{: j} \cdot\left(\mathbf{x}_{j}-\check{x}_{j}\right)\right) /\left(Y_{i:} \cdot \mathbf{J}_{: i}\right) .
\end{aligned}
$$

The quantity $\check{x}$, in the above formulae, is chosen to be mid $\mathbf{x}$.

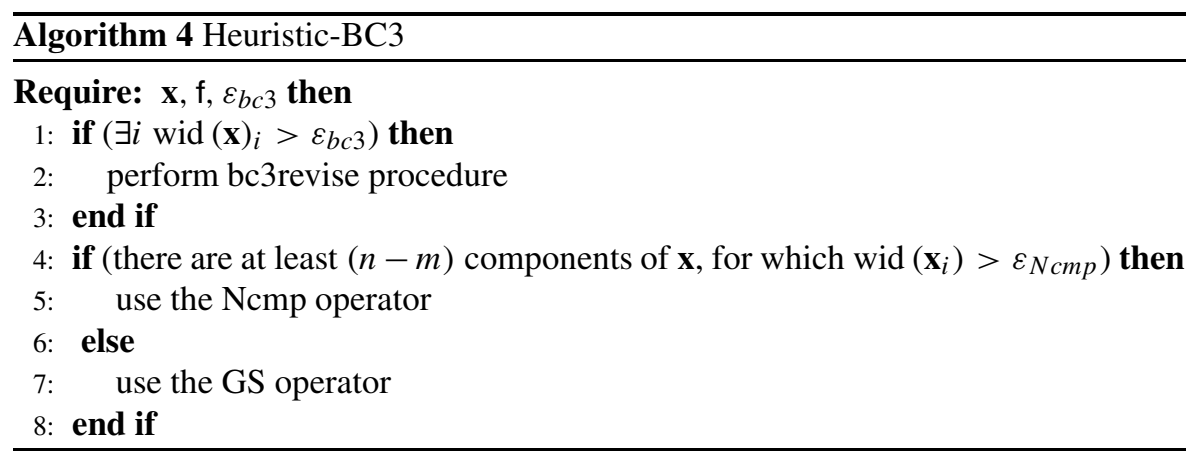




\section{Initial exclusion phase}

This tool, proposed by the author, has been described in [26, 28]. Before starting the B\&P method (i.e., Algorithm 1), we perform the procedure described by Algorithm 5 .

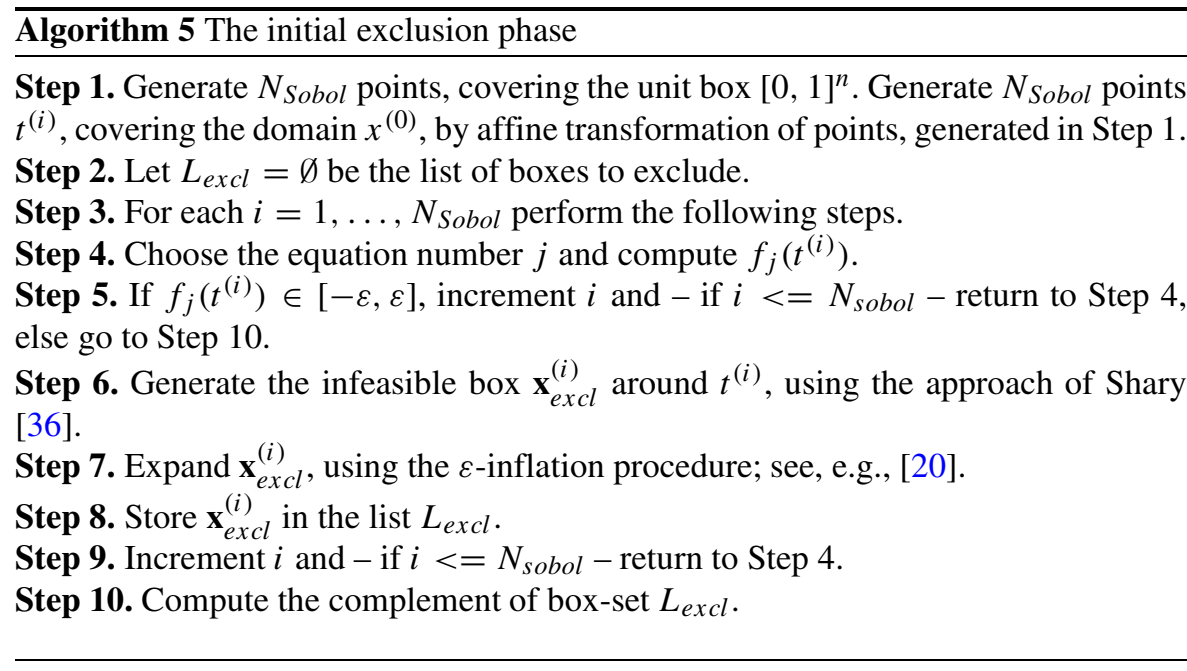

Algorithm 5 does not specify several important features:

- how to compute the elements of sequence $\left(t^{(i)}\right)$,

- which equation number $j$ to use for the point $t^{(i)}$; in [28] we used a roundrobin policy (the equation number $j=i \bmod m$ was used), which was quite an arbitrary assignment; an alternative is presented in one of the below paragraphs, - how to compute the complement of the created box-set $L_{\text {excl }}$ in $x^{(0)}$.

Computing the Sobol sequence is a relatively complex task, but there exist efficient and well-know algorithms (based on Gray code) and even open-source implementations (e.g., [8]). Surprisingly, a more difficult problem is computing the complement of the set of excluded boxes.

Using all equations for the exclusion Instead of arbitrary choosing a single equation for each $\left(t^{(i)}\right)$ in Algorithm 5, we can use all of the equations. Modification of the code is very simple and does not even require an additional loop.

For each point we start with the equation $f_{j}(x)=0$ for $j=1$. After realizing - in Step 7 - that we can no longer expand the box for this equation, we increment $j$ and proceed. The $\varepsilon$-inflation procedure is broken when $j$ becomes $m$ and no progress can be obtained for the last equation. Eventually, we choose $j$ for which the expanded box had the largest Lebesgue measure.

Additionally, we can expand the box even further if the problem is sparse. For each $j$ the excluded box, all variables $k$ such that $\frac{\partial f_{j}}{\partial x_{k}}=0$ in the whole domain, can be set to $\mathbf{x}_{k}=\mathbf{x}_{k}^{(0)}$. Let us call this technique sparsity-based expanding. 


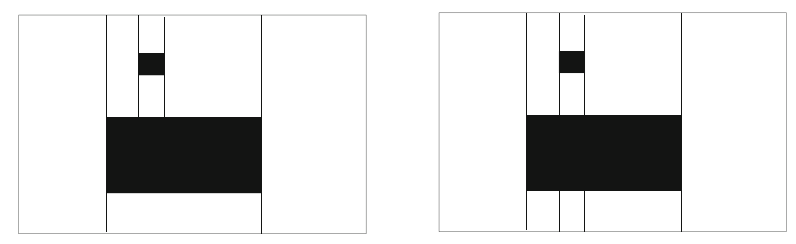

Fig. 3 Result of exclusion of two boxes - the larger or the smaller box is excluded first

Complement of a box-set There exist a well-known procedure - described by [20] - to compute the complement of a single box; we present it in Algorithm 6. The complement of the box-set can be computed by Algorithm 7, yet the procedure is inefficient. Not only, it does not parallelize well, but the resulting box-set may consist of too many boxes - see Fig. 3 (also described in [28]).

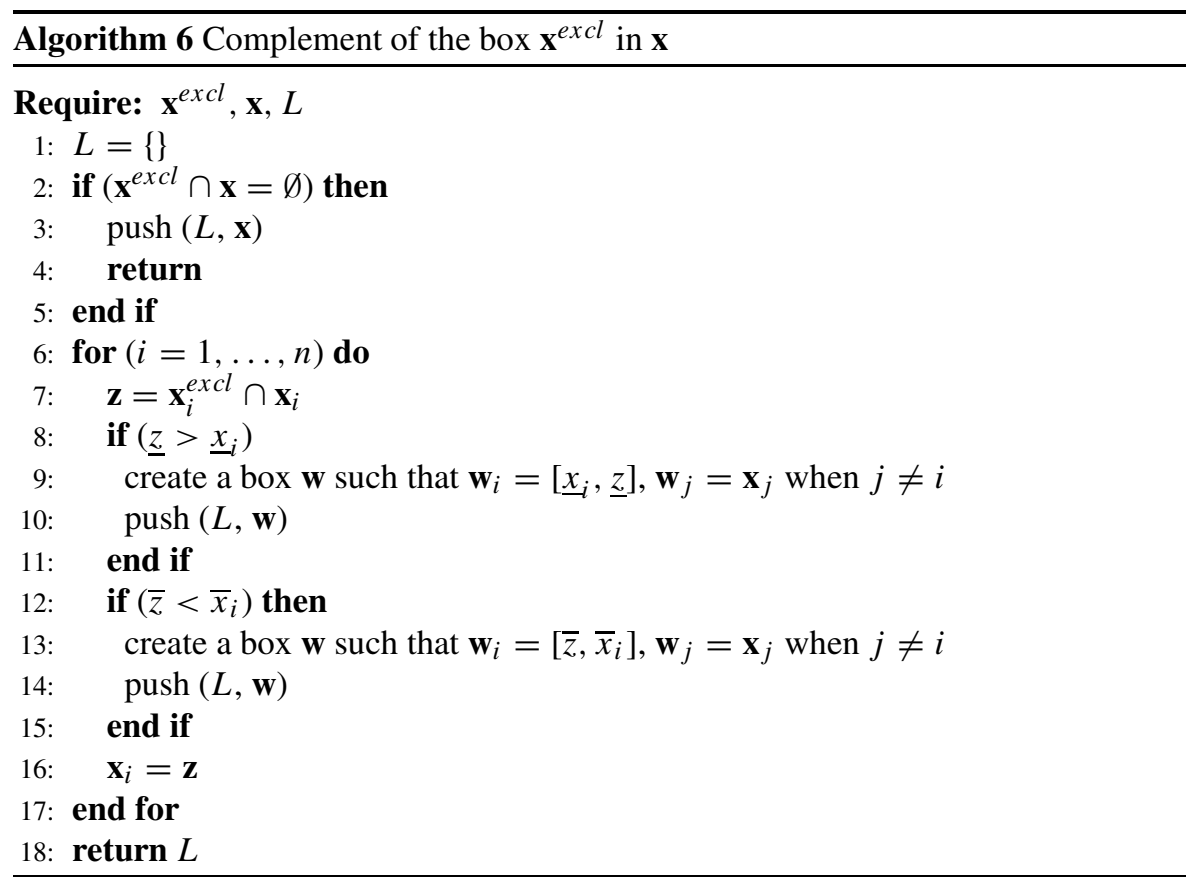

The problem is that we exclude the larger $\mathbf{x}^{\text {excl }}$ box from $\mathbf{x}$ first, but the larger excluded box can have a smaller intersection $\left(\mathbf{x}^{\text {excl }} \cap \mathbf{x}\right)$ with $\mathbf{x}$.

The simple improvement is to exclude from each box $\mathbf{x}$ the box that has the largest intersection with it - so we exclude different boxes from different parts of the domain at the same time, probably. Such a procedure can be parallelized, simply - using the task-parallelism model, which is used in TBB [2]. The procedure is described by Algorithm 8 


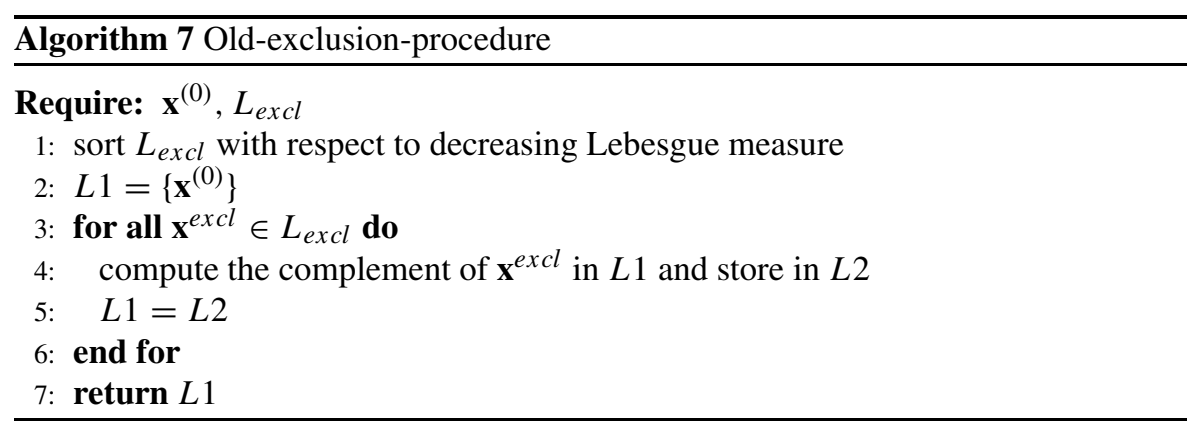

Yet another feature, used in the ultimate version of the algorithm, is not to exclude all boxes from $L_{\text {excl }}$. When we obtain the given number of boxes in $L-N_{\text {cutoff }}=$ 128 occurred to be a good choice - now boxes are not excluded, but x's from the remaining tasks are inserted into $L$ directly. This trick might seem peculiar, but it improves the performance, significantly.

Remark TBB templates tbb: :parallel_do and tbb: :parallel_do_feeder are very suitable for the implementation. The former allows a concurrent execution of a do...while loop, i.e., executing the same procedure for an unknown number (unlike parallel_for) of arguments. In our case: concurrent executions of Algorithm 8. And adding additional tasks is performed by a dedicated "feeder" object. Details can be found in [22] or, directly in TBB documentation [2].

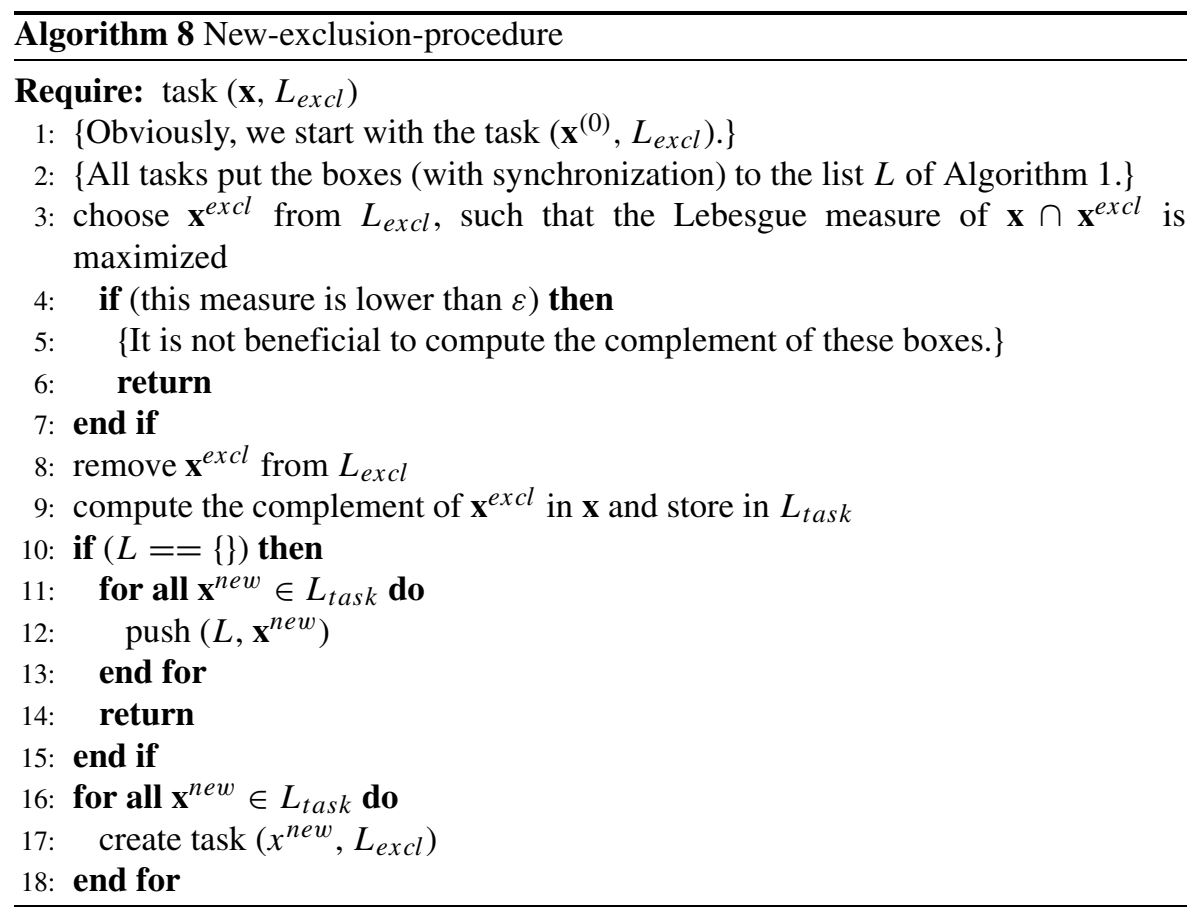




\section{Choosing the coordinate for bisection}

In [27] the problem of choosing the proper variable for bisection has been discussed. We emphasized the insufficiency of earlier approaches (see, e.g., [9]) and proposed the heuristic, described by Algorithm 9.

Its main idea was not to bisect the component that is the longest or has the maximal smear, but the one that will cause the resulting boxes to be convenient for the Newton operator to narrow. This led to the idea of choosing the component with the minimal magnitude. On the other hand, bisecting such components only, would result in loosing the convergence (also, it is not beneficial to have large differences between the component length, so if the difference between the longest and shortest component is too large, it is good to bisect the longest component). Hence we obtain a relatively complicated policy, trying to take into account all these facts. It is described by Algorithm 9.

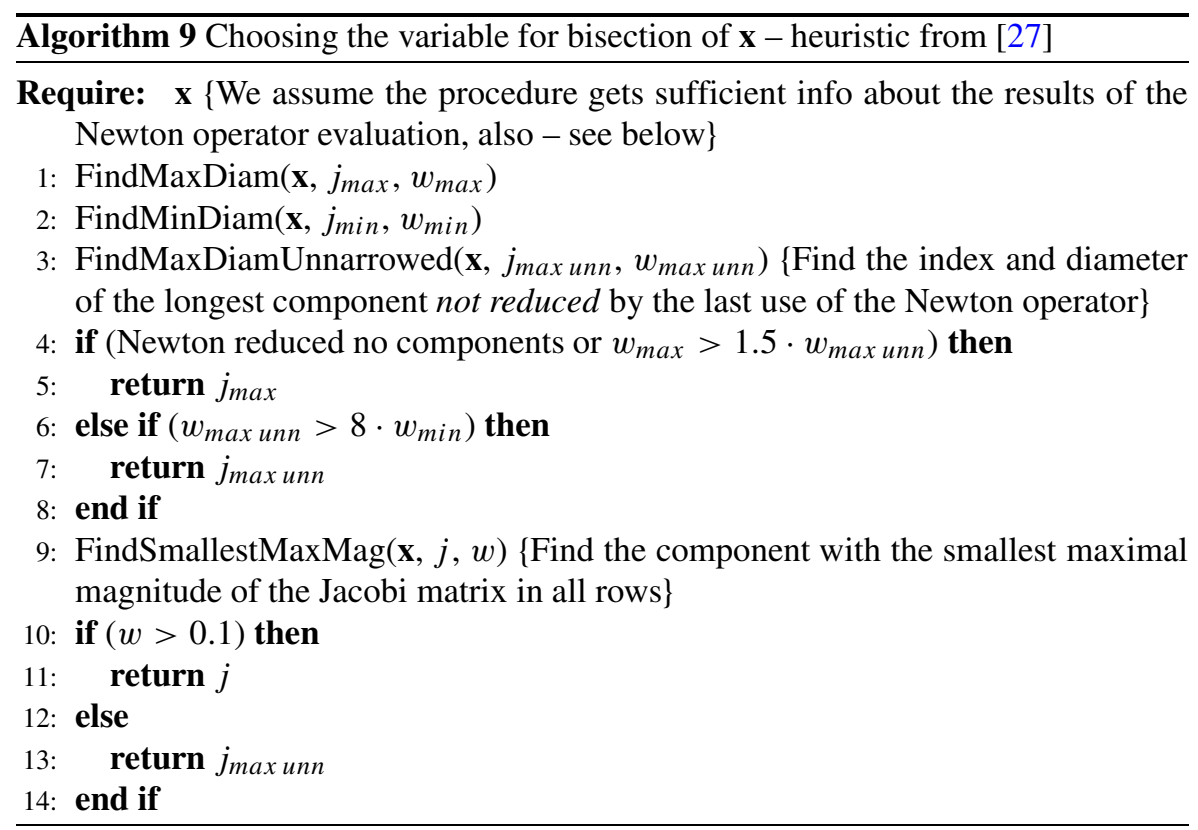

The algorithm was designed for underdetermined problems, but experiments in [27] have shown some improvements for well-determined problems, also.

A careful analysis shows, that the main reason of this improvement is avoiding to choose the components, narrowed by the Newton operator (by a narrowed component, we mean the one for which the operator had improved both bounds, i.e., $\mathbf{x}_{i}^{\text {new }} \subset$ int $\left.\mathbf{x}_{i}\right)$.

Should we choose the minimal magnitude components, indeed? For welldetermined problems, it is not beneficial, certainly - we should bisect components with the maximal magnitude as they have the largest influence on the overestimation 
of the solved functions. For underdetermined problems, the situation is more complicated. The above argument holds, but the component with the maximal magnitude is the one that should be narrowed by the Newton operator (for underdetermined problems not all components are narrowed to verify the solution existence!). Experiments with the MaxSumMag and MaxSmear heuristics (most of them are not presented due to lack of space, see also [27]) show a very poor performance of such policies for underdetermined problems.

Consequently, we propose to stick to choosing the maximal diameter for boxes that are not narrowed yet. For boxes where some components have already been narrowed, we can use the maximal sum magnitude heuristic, but only on unnarrowed components. It occurred that for smaller boxes, it is better to switch to the maximal diameter again (but, also, not bisecting the narrowed components).

For well-determined problems, the MaxSumMagnitude performs well, in general, but an exception to it is the Brent10 problem. Hence, we switch to MaxDiamUnnarrowed on occasions.

Details are given by the pseudocode in Algorithm 10.

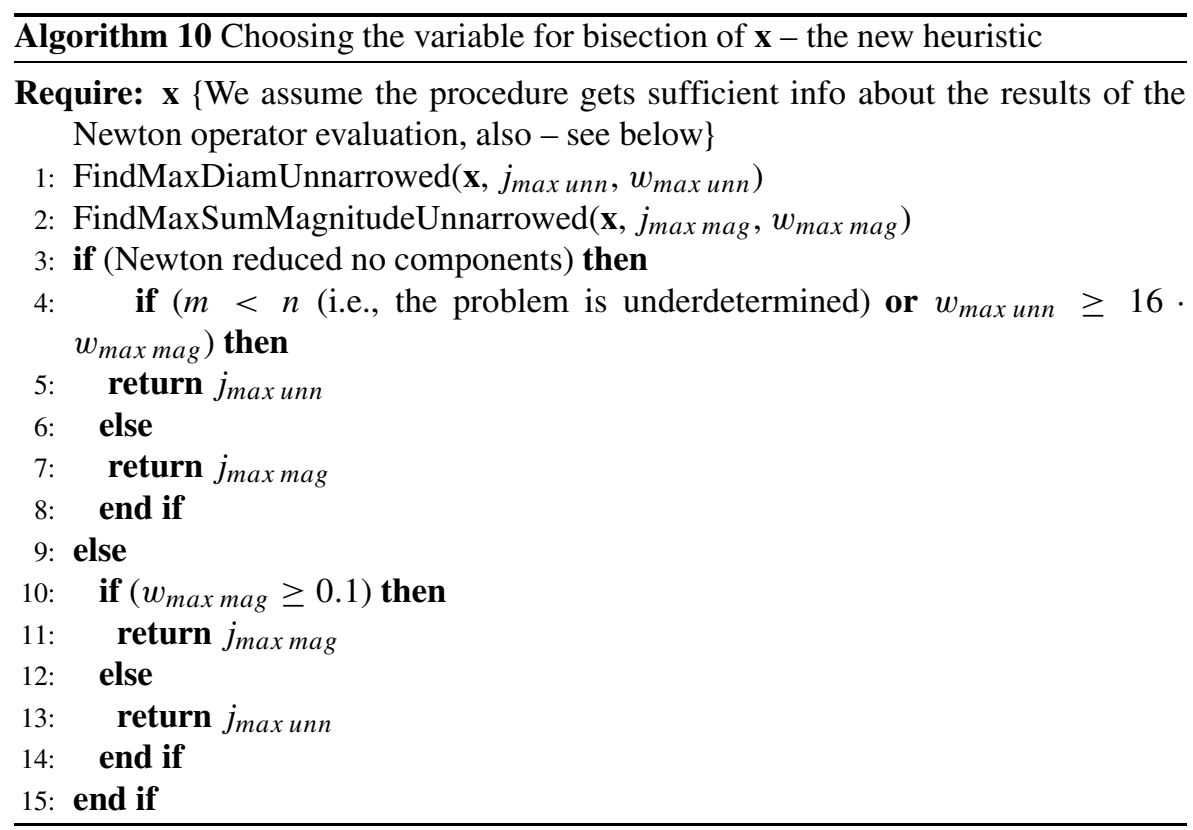

\section{Computational experiments}

Numerical experiments were performed on a computer with 4 cores (allowing hyperthreading), i.e., an Intel Core i7-3632QM with 2.2GHz clock. The machine ran under control of a 64-bit Manjaro 0.8.8 GNU/Linux operating system with the GCC 4.8.2, glibc 2.18 and the Linux kernel 3.10.22-1-MANJARO. 
The solver is written in $\mathrm{C}++$ and compiled using the GCC compiler. The $\mathrm{C}-\mathrm{XSC}$ library (version 2.5.3) [1] was used for interval computations. The parallelization ( 8 threads) was done with TBB 4.2, update 2 [2]. OpenBLAS 0.2.8 [3] was linked for BLAS operations.

We used 8 threads, on the 4 cores, which means hyper-threading was used on all cores. According to the author's experiences, it reduces the computation time by a factor of c.a. 0.9 with respect to having a single thread per core. Please note that parallelization does not affect the number of iterations, but the execution time only.

The following test problems were considered - four of them were underdetermined (Academic, Hippopede, Puma6, 5R planar) and five - well-determined (Box3, Bratu30, Brent10, Broyden16, Transistor).

The first of the underdetermined ones is a set of two equations - a quadratic one and a linear one - in five variables [13]. It is called the Academic problem.

$$
\begin{aligned}
& x_{1}^{2}+x_{2}^{2}+x_{3}^{2}+x_{4}^{2}+x_{5}^{2}-1.0=0, \\
& x_{1}+x_{2}+x_{3}+x_{4}+x_{5}=0, \\
& x_{1}, x_{2} \in[-1,1], x_{3} \in[-0.7,0.7], x_{4} \in[-0.8,0.8], x_{5} \in[-2,2] .
\end{aligned}
$$

Accuracy $\varepsilon=0.05$

The second one is called the Hippopede problem [25, 32] - two equations in three variables.

$$
\begin{aligned}
& x_{1}^{2}+x_{2}^{2}-x_{3}=0, \\
& x_{2}^{2}+x_{3}^{2}-1.1 x_{3}=0 . \\
& x_{1} \in[-1.5,1.5], x_{2} \in[-1,1], x_{3} \in[0,4] .
\end{aligned}
$$

Accuracy $\varepsilon=10^{-7}$ was set.

The third problem, called Puma, arose in the inverse kinematics of a $3 \mathrm{R}$ robot and is one of typical benchmarks for nonlinear system solvers [6].

$$
\begin{aligned}
& x_{1}^{2}+x_{2}^{2}-1=0, x_{3}^{2}+x_{4}^{2}-1=0, \\
& x_{5}^{2}+x_{6}^{2}-1=0, x_{7}^{2}+x_{8}^{2}-1=0, \\
& 0.004731 x_{1} x_{3}-0.3578 x_{2} x_{3}-0.1238 x_{1}-0.001637 x_{2}-0.9338 x_{4}+x_{7}=0, \\
& 0.2238 x_{1} x_{3}+0.7623 x_{2} x_{3}+0.2638 x_{1}-0.07745 x_{2}-0.6734 x_{4}-0.6022=0, \\
& x_{6} x_{8}+0.3578 x_{1}+0.004731 x_{2}=0, \\
& -0.7623 x_{1}+0.2238 x_{2}+0.3461=0, \\
& x_{1}, \ldots, x_{8} \in[-1,1] .
\end{aligned}
$$

In the above form it is a well-determined ( 8 equations and 8 variables) problem with 16 solutions that are easily found by several solvers. To make it underdetermined the last equation was dropped - as in [25] - resulting in 7 equations with 8 variables. Accuracy $\varepsilon=10^{-7}$ was set.

The fourth one is the inverse-kinematics problem of a planar redundant N-R manipulator, the effector of which should be placed in position $\left(1.0,1.0, \frac{\pi}{2}\right)$. We 
presented the problem in Section 1, already, but we repeat it here for the sake of completeness:

$$
\begin{aligned}
& \sum_{i=1}^{N} l_{i} \cdot \prod_{j=1}^{i} \cos \left(\sum_{k=1}^{j} x_{k}\right)-1=0, \\
& \sum_{i=1}^{N} l_{i} \cdot \prod_{j=1}^{i} \sin \left(\sum_{k=1}^{j} x_{k}\right)-1=0, \\
& \sum_{i=1}^{N} x_{i}-\frac{\pi}{2}=0, \\
& x_{i} \in\left[-\frac{\pi}{2}, \frac{\pi}{2}\right], \quad i=1, \ldots, N .
\end{aligned}
$$

We use this problem for $N=5, l_{i}=1, i=1, \ldots, 5$; the accuracy is set to $\varepsilon=2 \cdot 10^{-2}$.

The fifth problem is well-determined - it is called Box3 [6] and has three equations in three variables.

$$
\begin{aligned}
& \exp \left(-0.1 \cdot x_{1}\right)-\exp \left(-0.1 \cdot x_{2}\right)-x_{3} \cdot(\exp (-0.1)-\exp (-1.0))=0 \\
& \exp \left(-0.2 \cdot x_{1}\right)-\exp \left(-0.2 \cdot x_{2}\right)-x_{3} \cdot(\exp (-0.2)-\exp (-2.0))=0 \\
& \exp \left(-0.3 \cdot x_{1}\right)-\exp \left(-0.3 \cdot x_{2}\right)-x_{3} \cdot(\exp (-0.3)-\exp (-3.0))=0 \\
& x_{1}, x_{2} \in[-100.0,100.0], x_{3} \in[0.1,100.0]
\end{aligned}
$$

Accuracy $\varepsilon$ was set to $10^{-5}$.

The sixth problem is well-determined, also and very sparse; it is called Bratu [6].

$$
\begin{aligned}
& \frac{\exp \left(x_{1}\right)}{N+1}-2 x_{1}+x_{2}=0, \\
& x_{i-1}+\frac{\exp \left(x_{i}\right)}{N+1}-2 x_{i}+x_{i+1}=0, \quad i=2, \ldots, N-1, \\
& x_{N-1}+\frac{\exp \left(x_{N}\right)}{N+1}-2 x_{N}=0, \\
& x_{i} \in\left[-10^{8}, 20\right], \quad i=1, \ldots, N .
\end{aligned}
$$

We consider this problem for size $N=30$. Accuracy $\varepsilon=10^{-6}$ was set.

The seventh problem is called the Brent problem - it is a well-determined algebraic problem, supposed to be "difficult" [4].

$$
\begin{aligned}
& 3 x_{1} \cdot\left(x_{2}-2 x_{1}\right)+\frac{x_{2}^{2}}{4}=0 \\
& 3 x_{i} \cdot\left(x_{i+1}-2 x_{i}+x_{i-1}\right)+\frac{\left(x_{i+1}-x_{i-1}\right)^{2}}{4}=0, \quad i=2, \ldots, N-1, \\
& 3 x_{N} \cdot\left(20-2 x_{N}+x_{N-1}\right)+\frac{\left(20-x_{N-1}\right)^{2}}{4}=0 \\
& x_{i} \in\left[-10^{8}, 10^{8}\right], \quad i=1, \ldots, N .
\end{aligned}
$$


Presented results have been obtained for $N=10$; accuracy was set to $10^{-7}$.

The eight one is the well-known Broyden-banded system [6, 25].

$$
\begin{aligned}
& x_{i} \cdot\left(2+5 x_{i}^{2}\right)+1-\sum_{j \in J_{i}} x_{j} \cdot\left(1+x_{j}\right)=0, \quad i=1, \ldots, N, \\
& J_{i}=\{j \mid j \neq i \text { and } \max \{1, i-5\} \leq j \leq \min \{N, i+1\}\}, \\
& x_{i} \in[-100,101], \quad i=1, \ldots, N .
\end{aligned}
$$

In this paper we consider the case of $N=16$. The accuracy $\varepsilon=10^{-6}$ was set. And the last one we call "Transistor" is taken from [34]. It is a system of 9 equations in 9 variables:

$$
\begin{aligned}
& \left(1-x_{1} x_{2}\right) \cdot x_{3} \cdot\left(\exp \left(x_{5} \cdot\left(g_{1 k}-g_{3 k} \cdot 10^{-3} \cdot x_{7}-g_{5 k} \cdot 10^{-3} \cdot x_{8}\right)\right)-1\right)+ \\
& -g_{5 k}+g_{4 k} \cdot x_{2}=0, \quad k=1, \ldots, 4, \\
& \left(1-x_{1} x_{2}\right) \cdot x_{4} \cdot\left(\exp \left(x_{6} \cdot\left(g_{1 k}-g_{2 k}-g_{3 k} \cdot 10^{-3} \cdot x_{7}+g_{4 k} \cdot 10^{-3} \cdot x_{9}\right)\right)-1\right)+ \\
& -g_{5 k} \cdot x_{1}+g_{4 k}=0, \quad k=1, \ldots, 4 \\
& x_{1} \cdot x_{3}-x_{2} \cdot x_{4}=0 . \\
& x_{i} \in[0,10], \quad i=1, \ldots, 9 .
\end{aligned}
$$

The matrix of $g_{m k}$ parameters can be found, e.g., in [34] and [33]. Accuracy $\varepsilon=10^{-8}$ was used in our experiments.

The following notation is used in the tables:

- fun.evals, grad.evals, Hesse evals - numbers of functions evaluations, its gradients and Hesse matrices evaluations (in the interval automatic differentiation arithmetic),

- bisecs - the number of boxes bisections,

- preconds - the number of preconditioning matrix computations (i.e., performed Gauss-Seidel steps),

- $\quad$ bis.Newt, del.Newt - numbers of boxes bisected/deleted by the Newton step,

- Sobol excl. - the number of boxes to be excluded generated by the initial exclusion phase,

- Sobol resul. - the number of boxes resulting from the exclusion phase, i.e., the size of the box-set $L$ to be considered by the B\&P method,

- bc3 - the number of calls of the consistency enforcing algorithm - Algorithm 3,

- bc3.rev. - the number of "first-level" calls (i.e., not counting the recursive ones) of "left_narrow" and "right_narrow" procedures,

- del.bc3 - the number of boxes deleted by consistency enforcing,

- q.solv - the number of interval quadratic equations the algorithm was trying to solve,

- q.del.delta - the number of boxes deleted, because the discriminant of the quadratic equation was negative,

- q.del.disj. - the number of boxes deleted, because the solutions of a quadratic equation were disjoint with the original box,

- q.bisecs - the number of boxes bisected by the quadratic equations solving procedure, 
- pos.boxes, verif.boxes - number of elements in the computed lists of boxes containing possible and verified solutions,

- Leb.pos., Leb.verif. - total Lebesgue measures of both sets,

- time - computation time in seconds.

The ultimate table - Table 14, showing results for the currently most efficient algorithm version - has two additional rows, describing speedups with respect to two reference versions:

- $\quad \mathrm{sp}($ basic) - with respect to version "basic+BLAS" (see below for the description of both names),

- $\quad$ sp(PPAM) - with respect to version "PPAM2011".

We present results for the following algorithm versions:

- basic - for each box we compute the Jacobi matrix and use the interval GaussSeidel step with inverse-midpoint preconditioner; bisection over the variable with maximal diameter; no additional tools,

- basic+BLAS - as above, but the inverse-midpoint preconditioner is computed approximately and BLAS procedures are applied for matrix operations,

- PPAM2011 - the version presented in [27],

- PPAM2011+BC3( $\left.\varepsilon_{b c 3}\right)$,

- PPAM2011+QH - the version presented in [27], with the Hansen's quadratic test, but no Sobol exclusion phase; see [29],

- PPAM2011+Sobol $(k)$ - the version presented in [28], but with the new complement computing algorithm,

- PPAM2011+BC3 $\left(\varepsilon_{b c 3}\right)+\mathrm{QH}$, PPAM2011+Sobol $(k)+\mathrm{BC} 3\left(\varepsilon_{b c 3}\right)$, etc. - various combinations of the used tools.

Tables $1,2,3,4,5,6,7,8,9,10,11$ and 12 contain results for several simple versions of the algorithm, using many variants of the used tools. The two ultimate tables - Tables 13 and 14 contain the experiments for versions that -according to previous experiments - occurred to be most promising (see the analysis in the next section).

Remark 1 Please note that Table 1 contains two sets of results - the additional row ("BLAS-time") presents the computation times of the "basic+BLAS" algorithm version. This is done to save space. Other quantities, i.e., numbers of function evaluations, gradients, etc. are not presented for this version as they are very similar to results for the "basic" version. Very similar, but not identical - minor differences can be observed for problems Hippopede, 5R planar and Box3. Details are available form the author upon request.

Remark 2 Please note, results of the exclusion phase in its current version are not deterministic. The number in the filed "Sobol resul." may vary by a small factor and also computational time may be a few seconds higher or lower (also the number of 


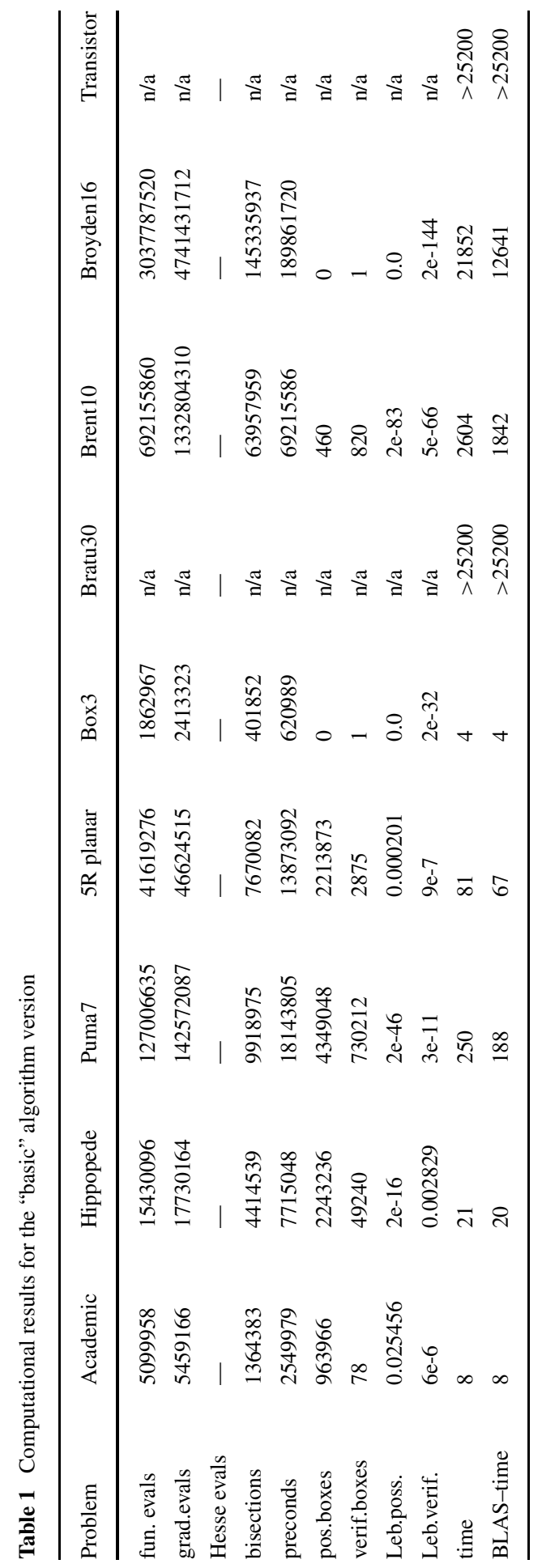




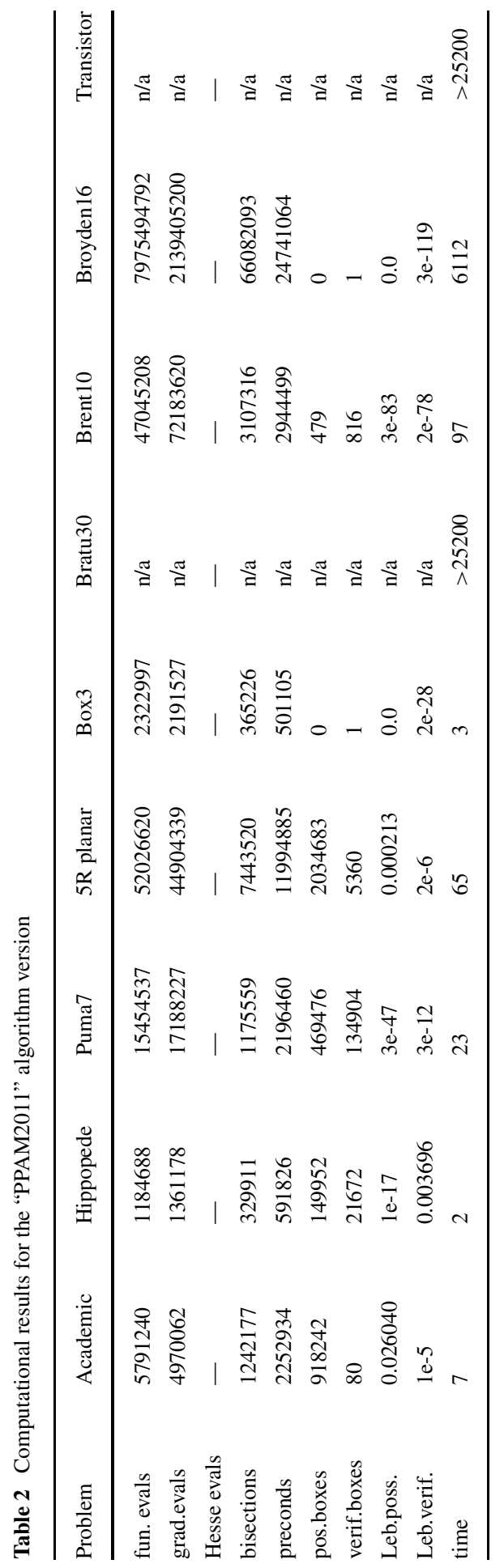




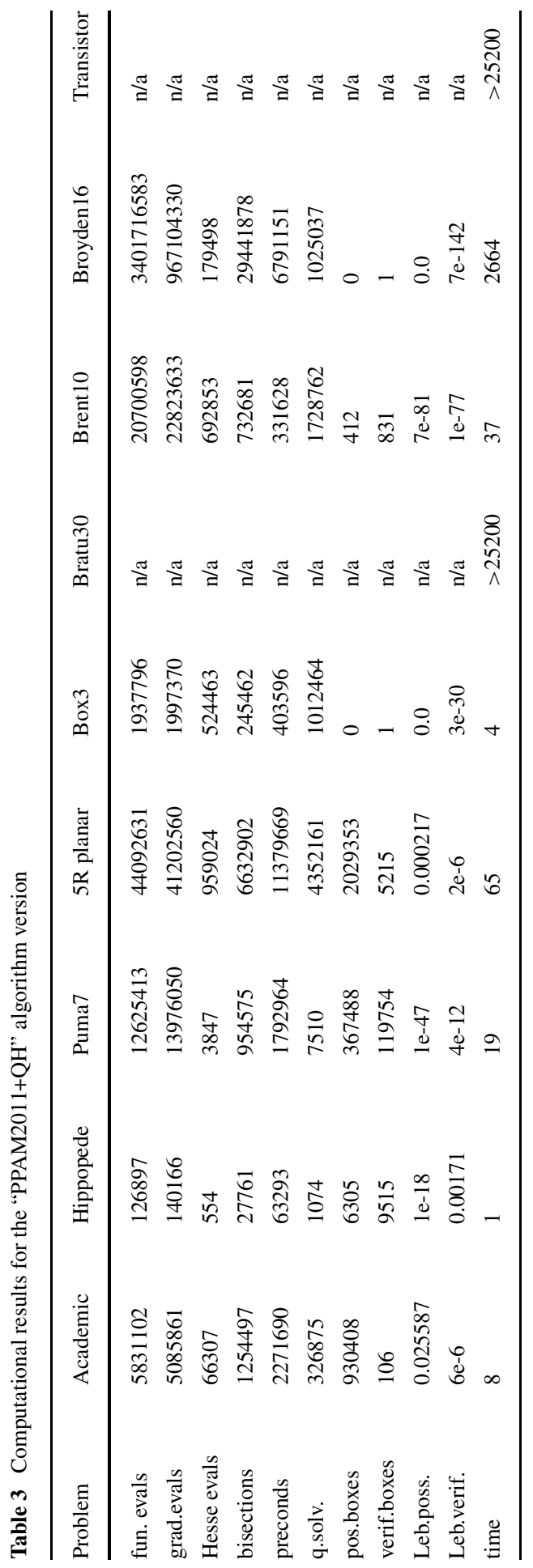




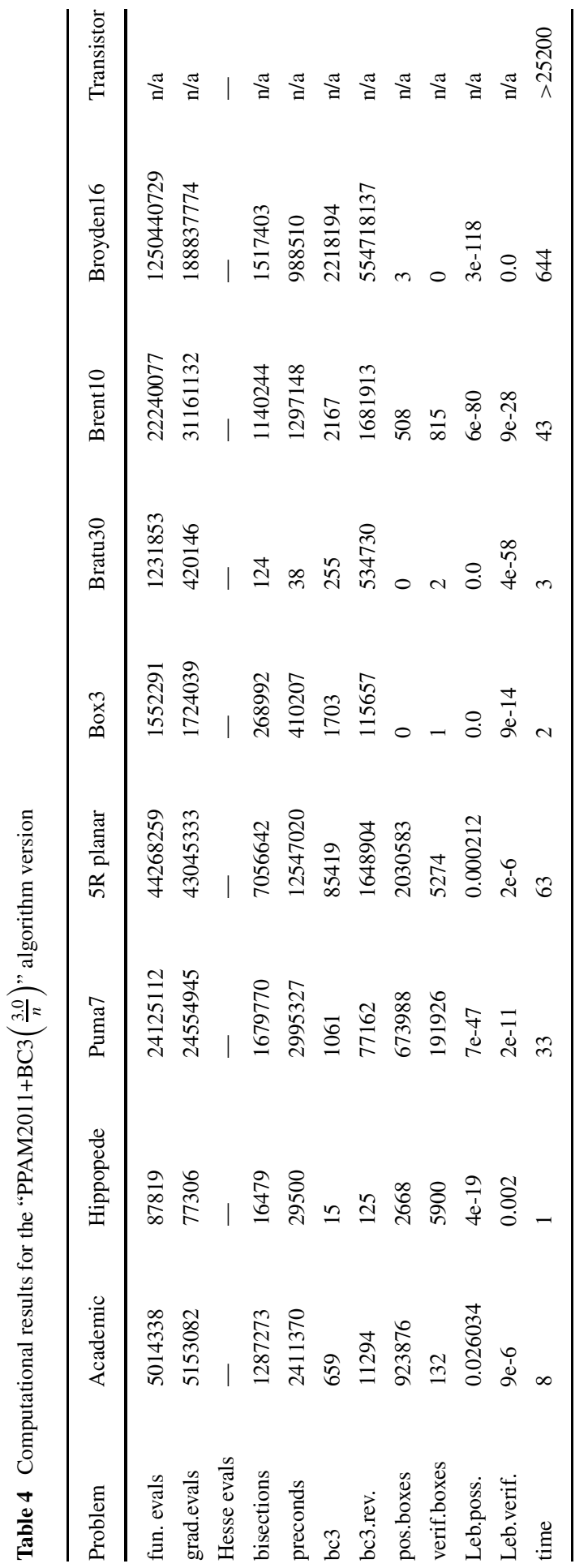




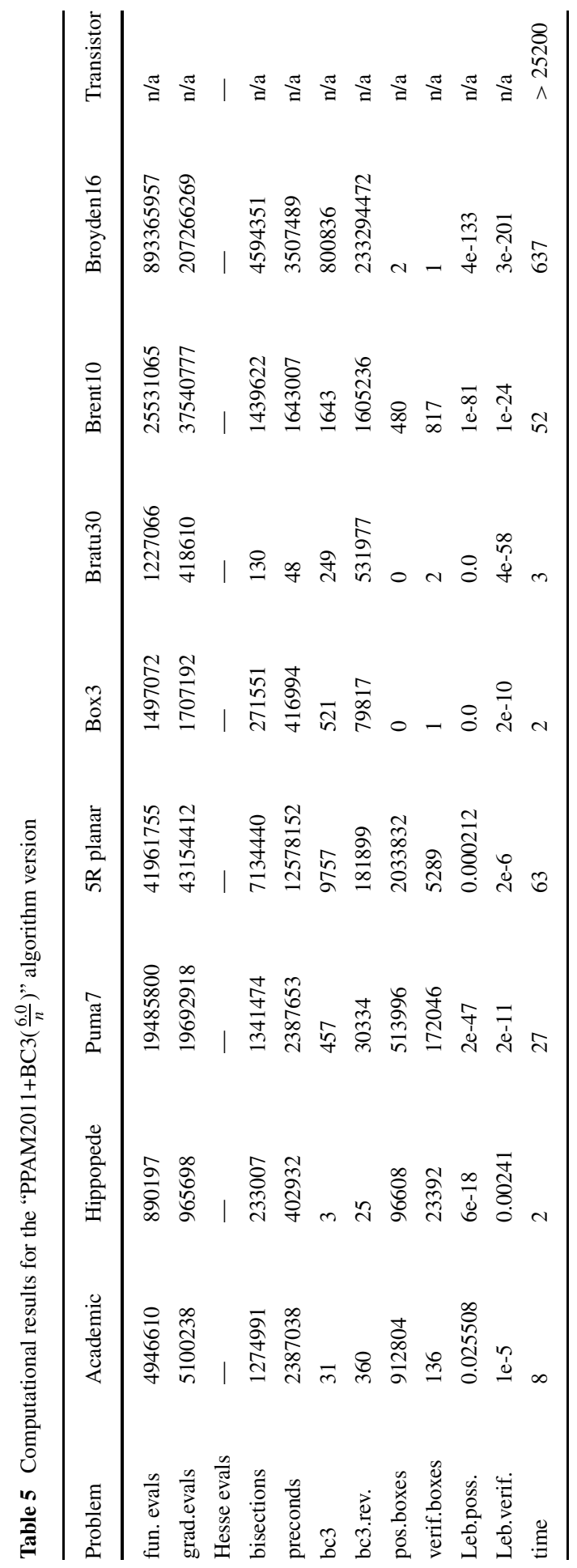




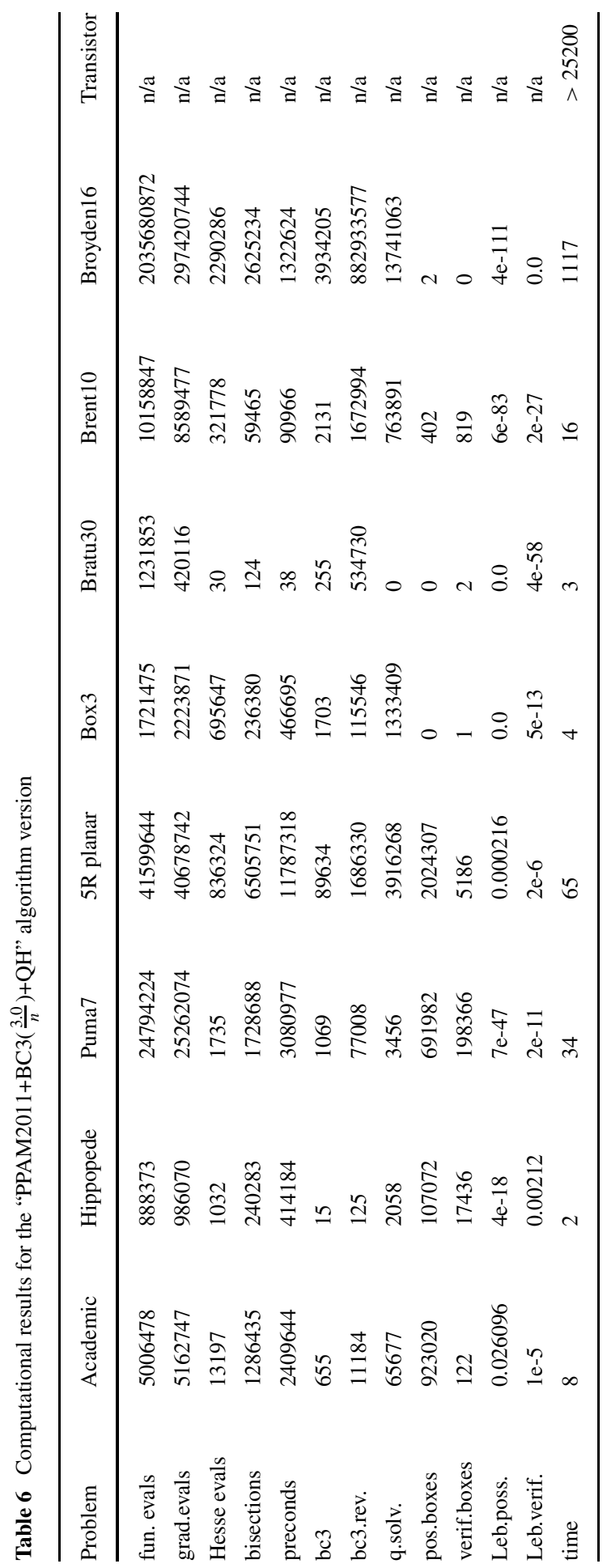




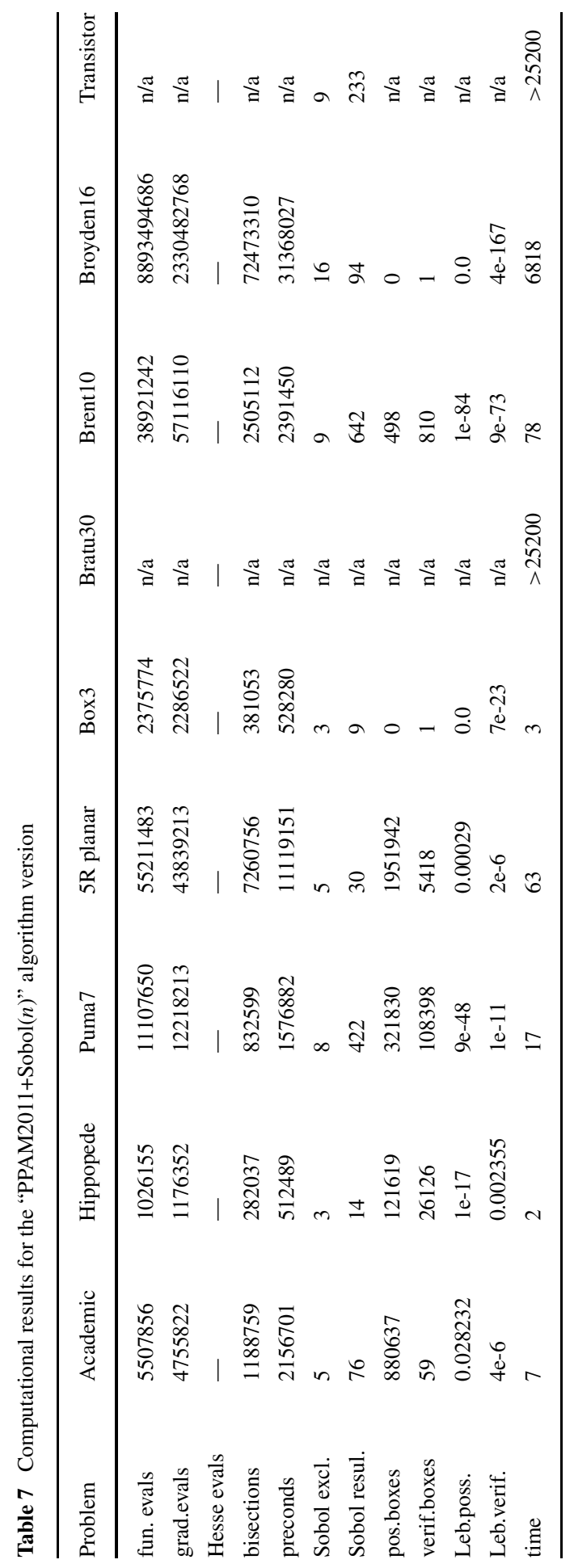




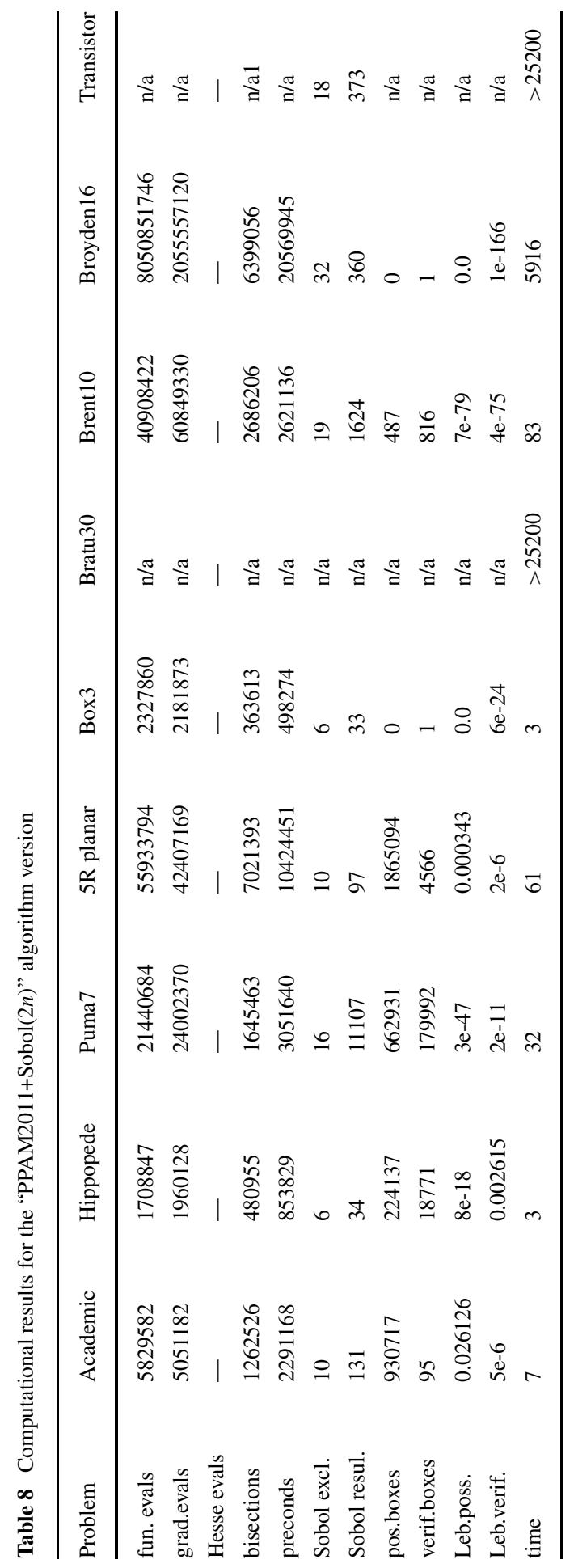




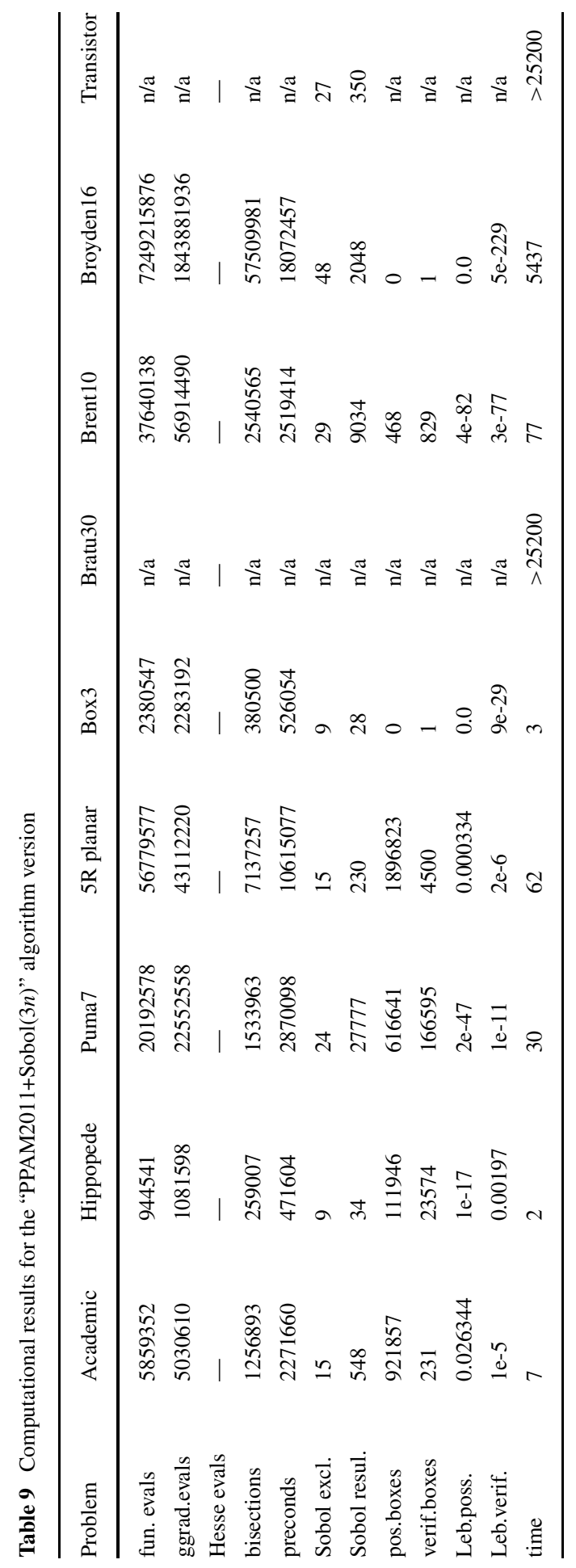




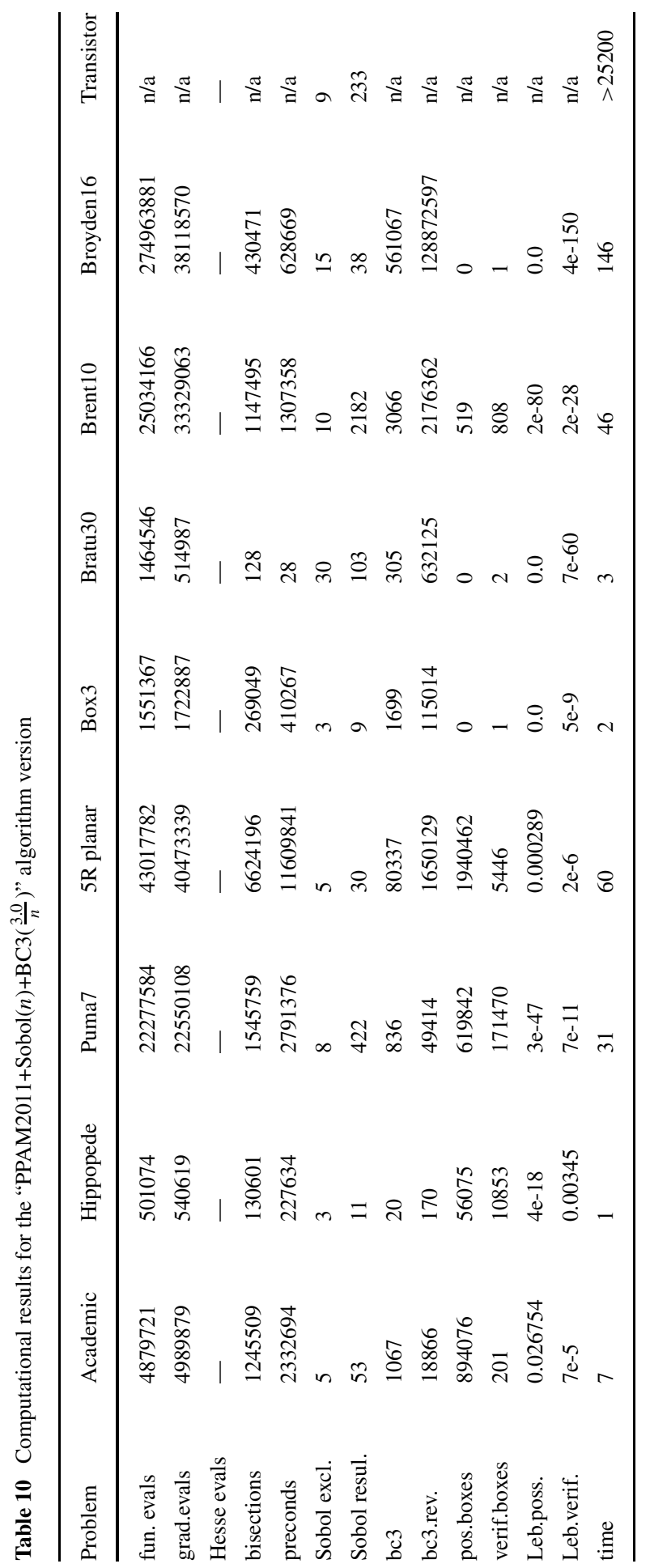




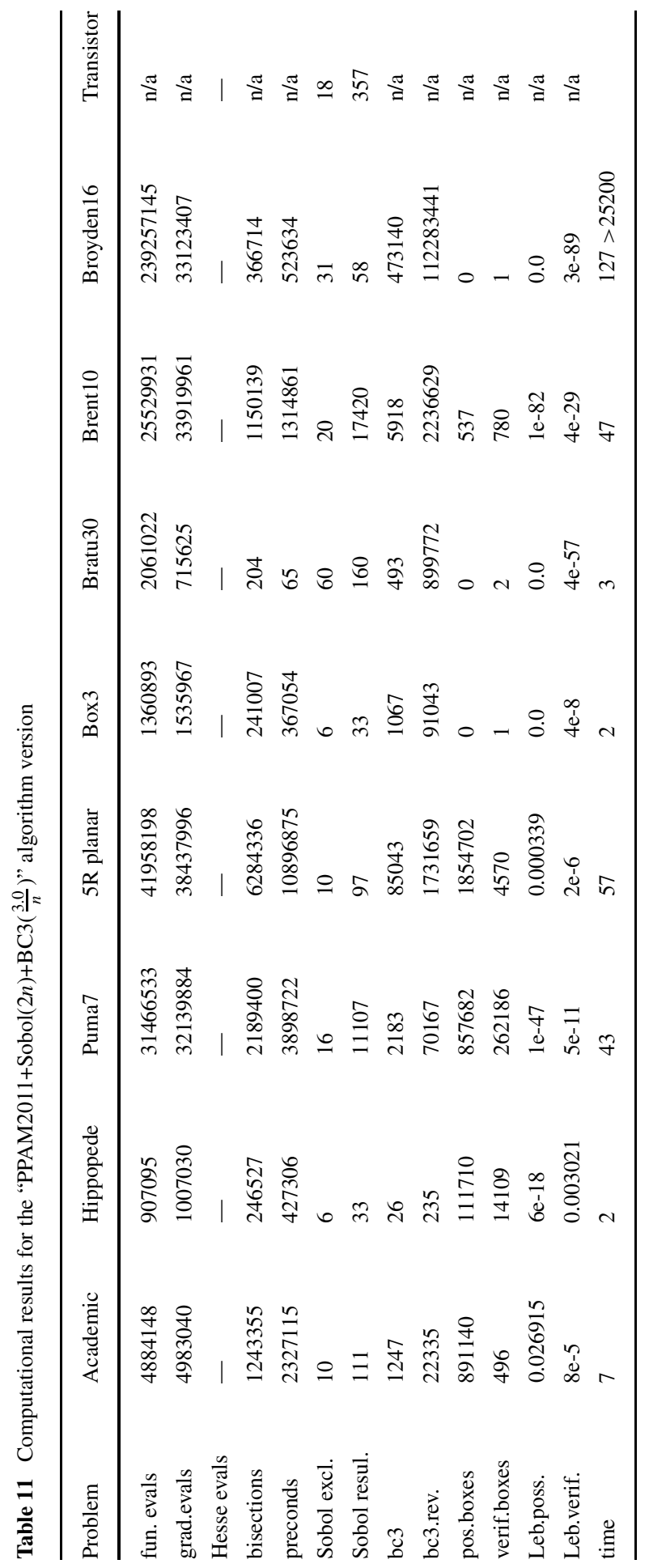




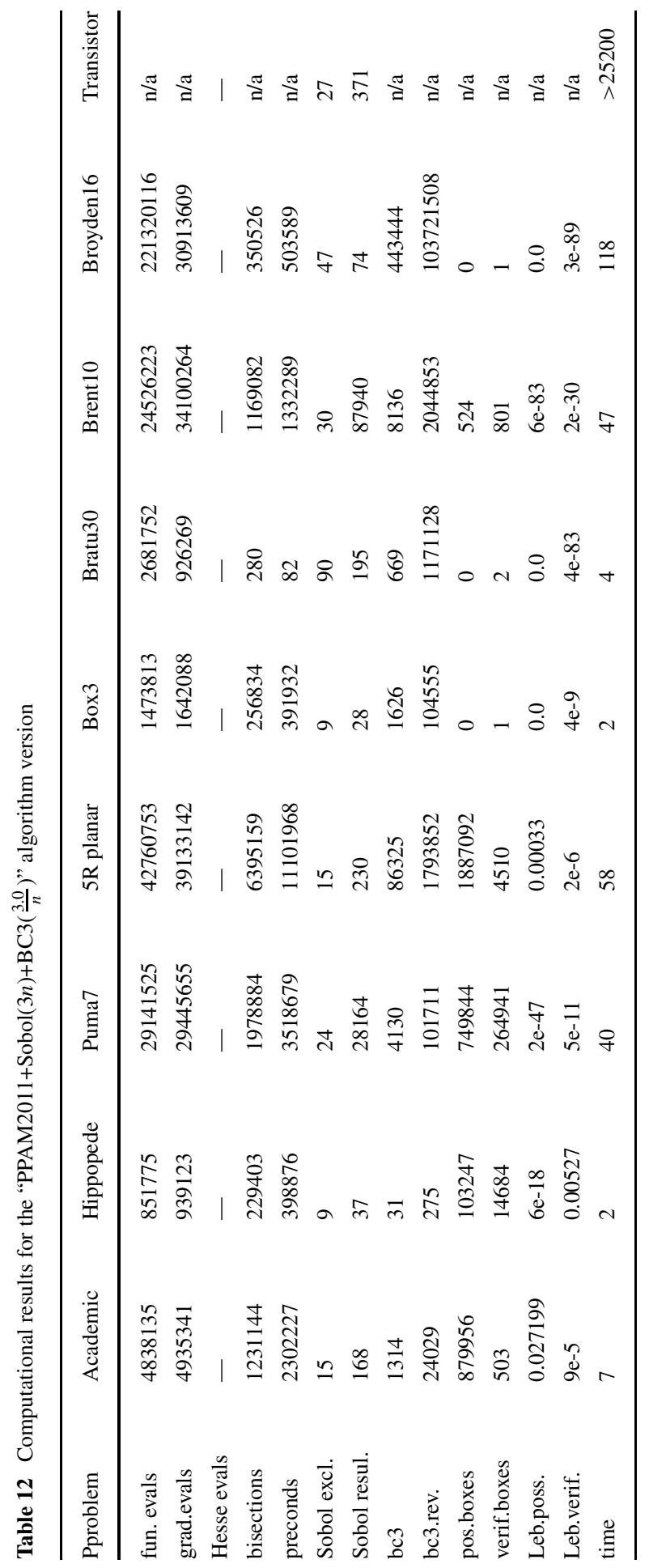




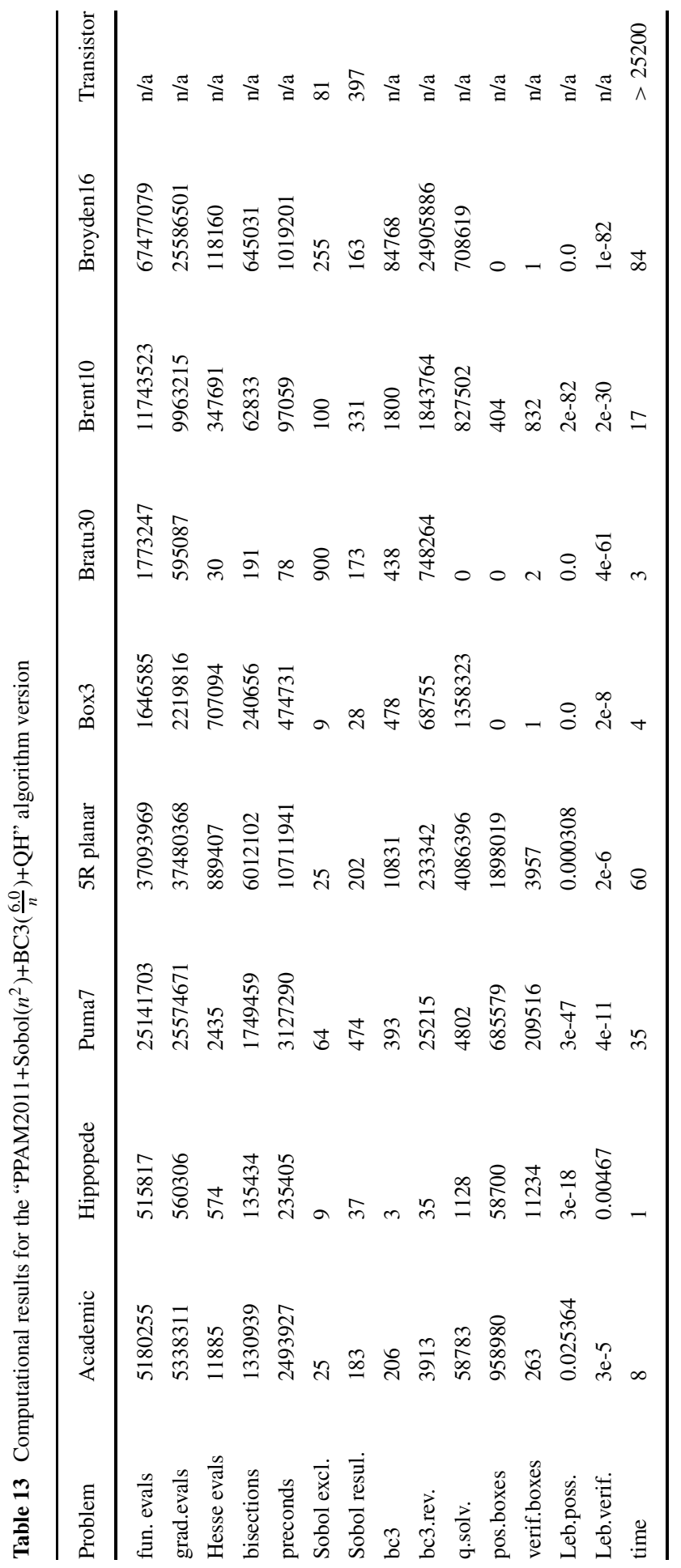




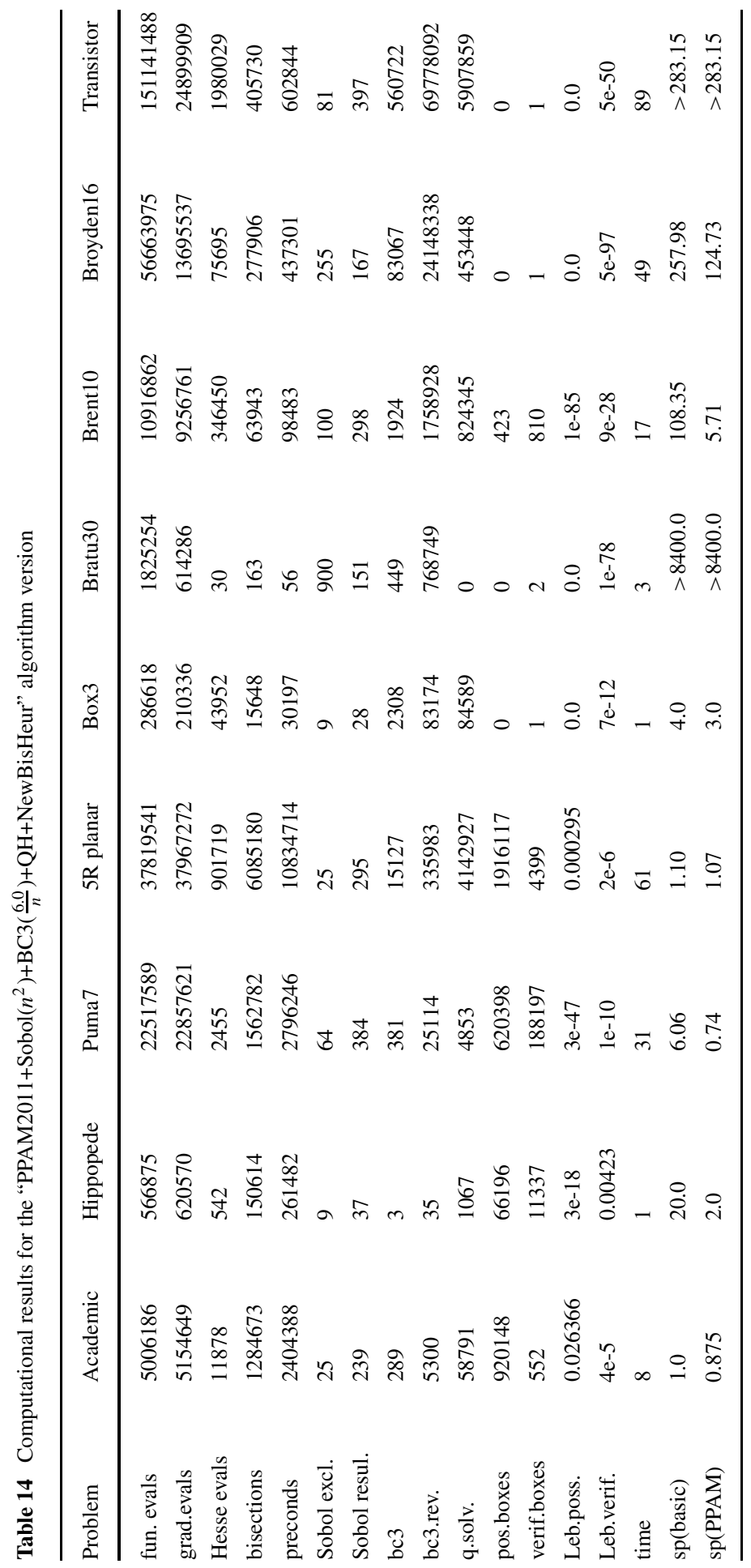


iterations, etc., obviously). We do not emphasize it in the tables (nor present any statistical analysis of the phenomenon) as the uncertainty is minor.

\section{Selected results obtained for Realpaver}

For comparison, we present results for three test problems, obtained using another solver, Realpaver [7]. It is one of mature interval solvers that can be considered current state-of-the-art [17].

$5 R$ planar. For this underdetermined problem, Realpaver required 17 minutes (for Bisection precision $=2.0$, much less accurate than the presented solver) and did not cover the whole solution set ("Property: non reliable process (some solutions may be lost)"). This result was far worse than ours.

Brent10. For this well-determined problem Realpaver has found all solutions (1065) in 55 seconds, but only when -number 2000 was enforced. For default settings, it returned after 46 seconds with an incomplete list of boxes. Again, a worse result than for the presented solver.

Transistor. For this problem, Realpaver outperformed our solver. For the settings proposed by Realpaver authors (trisection and using weak 3B consistency; the benchmark is pre-defined in the configuration files of this solver), the solver requires 13 seconds, but does not verify the unique solution, but returns with a cluster of 3 boxes. For the default settings, Realpaver verifies the unique solution, but it takes 30 seconds - still a better result than our solver.

\section{Analysis of results}

Results, presented in Section 6 show that the performance impact of various tools may vary to the high extent. Using a single tool (initial exclusion phase, box consistency enforcing or Hansen's quadratic test) often improves the performance of the "PPAM2011" algorithm version. But applying two of the successful operations may result in far a smaller improvement or even in a slowdown. Apparently, some expensive tools might "redundant" when used together with other ones.

In particular, applying the Hansen's quadratic test improves the performance of the "PPAM2011" algorithm for problems Hippopede, Puma7 and Broyden16 (see Tables 2 and 3). But when we apply both $\mathrm{BC} 3(3.0 / n)$ procedure and the quadratic test, results will be worse than for BC3 only (see Tables 4 and 6). Probably, the quadratic test (that requires Hesse matrix computation!) is applied for some boxes that could be reduced by the $\mathrm{BC} 3$ procedure, but it is difficult to verify this conjecture.

On the other hand, the Hansen's quadratic test improves the performance of all versions for the Brent10 problem. 
In general, applying the $\mathrm{BC} 3$ procedure seems very worthwhile (improvements are dramatic for problems Brent10, Broyden16 and - particularly - Bratu30 that cannot be solved in a reasonable time without using the consistency operator), yet it is completely useless for the Puma7 problem (reasons for this behavior remain to be determined). The improvement for Box 3 problem is minor, but irrefutable. Improvements for problems Academic and 5R-planar are minor (or none), but this seems to be related to the fact that these problems are underdetermined with the difference between the number of variables and equations of more than one ( 3 and 2 , respectively; see below).

For two problems - Box3 and Transistor - it occurred to be crucial, to choose the proper coordinate for bisection, i.e., to use a heuristic related to MaxSmear, e.g., Algorithm 10.

The Transistor problem For this problem, only one algorithm version was able to provide the results. It was the most efficient version - results are presented in Table 14. Other experiments, not presented in this paper, show that for the Transistor problem, useful are only the following algorithm versions have the following properties, mutually:

- they use box-consistency,

- they use Algorithm 10.

The ultimate version gives the correct solution (the single box, guaranteed to contain the solution) in 89 seconds. Solvers, presented in [33] require 2359.5 seconds for the version tuned for this specific problem and 135099 for a more general version and in [16] - 444 seconds. These experiments have been performed on a Sun Ultra-2 running Solaris; according to [16], the clock frequency was $166 \mathrm{MHz}$.

Our results are much better, but they are obtained on a far stronger machine, also. For using Realpaver, the correct solution was computed far quicker, on our machine -30 seconds.

Apparently, the use of hull-consistency (that was used in [16] and also is incorporated in Realpaver) is pretty worthwhile. Unfortunately, hull-consistency enforcing is not easy to implement, especially in multithreaded environments. It requires complicated expression tree building and each thread should be able to traverse the tree (forward and backward) independently (intervals of values of respective quantities in the tree must be thread-specific). Still, the effort has to be done.

Underdetermined vs well-determined problems It is worth noting that tuning the algorithm for underdetermined problems occurred to be much harder than for welldetermined ones. The Hippopede problem seems to be particularly "capricious" results change rapidly for minor changes of algorithm features.

If the dimension of the solution set is higher than one, i.e., the difference between the number of variables and equations is higher than one, then tuning the algorithm does not have a significant impact on the performance. Comparing all tables shows that for such problems all algorithm versions perform similarly; it seems the $\varepsilon$ we have to choose for such problems to stop in a reasonable time is so large, that specific features of various algorithm versions do not "have time" to affect the performance 
(or the time necessary to process all boxes containing solutions is too long). Experiments presented in the paper - problems Academic and 5R planar - but also in previous ones - problems Puma6 and Rheinboldt, see, e.g., [25-29] are consistent with this observation.

Also, underdetermined problems seem to require different policies for bisection than well-determined ones. In particular, heuristics based on smear computation, like MaxSmear, MaxSumMagnitude (e.g., [9]) perform particularly bad on them. It seems to be caused by the fact that not all of the components are going to be narrowed for underdetermined problems, but only the ones with the high smear, so the other components should be bisected, instead. If some components have been narrowed by the Newton operator, we should not bisect them (MaxSumMagnitudeUnnarrowed), but for boxes not narrowed yet, heuristics based on smear and magnitude cannot be applied at all. On the other hand, components with small smear and magnitude have minor impact on the system. So, it seems, bisecting the longest edge is the best solution and that is what we do in Algorithm 10.

The currently-best version Overall, the version that performs best, currently, occurred to be the one with the following features:

- the initial exclusion phase with $n^{2}$ Sobol points generated, sparsity-based expanding (see Section 4 ) and $N_{\text {cutoff }}=128$,

- Algorithm 4 is used to decide whether to use BC3 or not, $\varepsilon_{b c 3}=\frac{6.0}{n}$,

- the variable for bisection chosen by heuristic, described in Algorithm 10.

Results for this version are presented in Table 14.

\section{Conclusions}

Interval branch-and-prune solvers can use a great deal of tools to narrow and discard boxes. In this paper, the usefulness of some of them (proposed by the author and by other researchers) has been investigated. A proper heuristic to choose and parameterize the tools has been proposed.

In particular, we presented a novel initial exclusion phase and a new policy to choose the variable for bisection. This policy distinguishes underdetermined and well-determined problems, which seems another important novelty.

As test examples show, the proposed algorithm performs well and is successful for some hard problems (e.g., the Brent problem).

Comparison with the Realpaver solver imply that our solver can outperform it for underdetermined and non-typical (e.g., singular or ill-determined) problems, but performs much worse for the Transistor problem.

It is probably far from optimal and further research is going to be performed - in particular, applying machine learning techniques to self-tune the algorithm.

The source code of the presented version of the algorithm (and hopefully further versions) is going to be available at the author's page: https://www.researchgate.net/ profile/Bartlomiej_Kubica?ev=hdr_xprf. 
Future research Interesting results might be obtained, by applying AI methods to self-tune the heuristics. Up to now, the only paper investigating such an approach (but in a very limited version) is [15].

Also, hull consistency enforcing must be investigated (as we indicated earlier) this procedure appears very useful in experiments performed by other researchers. So are the acceleration tools, proposed by Kolev [21].

Acknowledgements The author would like to thank Adam Woźniak and Sergey P. Shary for inspiring discussions.

Also, the author is grateful to the reviewers for helping to improve the paper by their valuable suggestions.

Open Access This article is distributed under the terms of the Creative Commons Attribution License which permits any use, distribution, and reproduction in any medium, provided the original author(s) and the source are credited.

\section{References}

1. C++ extended scientific computing library. http://www.xsc.de (2013)

2. Intel Threading Building Blocks. http://www.threadingbuildingblocks.org (2013)

3. OpenBLAS library. http://xianyi.github.com/OpenBLAS/ (2013)

4. Difficult benchmark problems. http://www-sop.inria.fr/coprin/logiciels/ALIAS/Benches/node6.html (2014)

5. GNU linear programming kit.http://www.gnu.org/software/glpk/ (2014)

6. Non-polynomial nonlinear system benchmarks. https://www-sop.inria.fr/coprin/logiciels/ALIAS/ Benches/node2.html (2014)

7. Realpaver. nonlinear constraint solving \&amp; rigorous global optimization. http://pagesperso.lina. univ-nantes.fr/info/perso/permanents/granvil/realpaver/ (2014)

8. Sobol sequence generator. http://web.maths.unsw.edu.au/fkuo/sobol/ (2014)

9. Beelitz, T., Bischof, C.H., Lang, B.: A hybrid subdivision strategy for result-verifying nonlinear solvers. Tech. Rep. 04/8, Bergische Universitiät Wuppertal (2004)

10. Benhamou, F., Goualard, F., Granvilliers, L., Puget, J.F.: Revising hull and box consistency. In: International Conference on Logic Programming, pp. 230-244. The MIT Press (1999)

11. Benhamou, F., McAllester, D., Hentenryck, P.V.: CLP(intervals) revisited. In: Logic Programming, Proceedings of the 1994 International Symposium, pp. 124-138. The MIT Press (1994)

12. van Emden, M.H.: Computing functional and relational box consistency by structured propagation in atomic constraint systems. arXiv:preprint cs/0106008 (2001)

13. Goldsztejn, A., Jaulin, L.: Inner and outer approximations of existentially quantified equality constraints. Lect. Notes Comput. Sci. 4204, 198-212 (2006)

14. Goualard, F.: On considering an interval constraint solving algorithm as a free-steering nonlinear Gauss-Seidel procedure. In: Proceedings of the 2005 ACM Symposium on Applied Computing, SAC '05, pp.1434-1438, ACM, New York, NY, USA (2005). URL http://doi.acm.org/10.1145/1066677. 1067004

15. Goualard, F., Jermann, C.: A reinforcement learning approach to interval constraint propagation. Constraints 13(1-2), 206-226 (2008)

16. Granvilliers, L., Benhamou, F.: Progress in the solving of a circuit design problem. J. Glob. Optim. 20(2), 155-168 (2001)

17. Granvilliers, L., Benhamou, F.: Algorithm 852: Realpaver: an interval solver using constraint satisfaction techniques. ACM Trans. Math. Softw. (TOMS) 32(1), 138-156 (2006)

18. Hansen, E., Walster, W.: Global Optimization Using Interval Analysis. Marcel Dekker, New York (2004)

19. Ishii, D., Goldsztejn, A., Jermann, C.: Interval-based projection method for under-constrained numerical systems. Constraints 17(4), 432-460 (2012) 
20. Kearfott, R.B.: Rigorous Global Search: Continuous Problems. Kluwer, Dordrecht (1996)

21. Kolev, L.V.: An improved interval linearization for solving nonlinear problems. Numeri. Algorithm. 37(1-4), 213-224 (2004)

22. Kubica, B.J.: Intel TBB as a tool for parallelization of an interval solver of nonlinear equations systems. Tech. Rep. 09-02, ICCE WUT (2009)

23. Kubica, B.J.: Performance inversion of interval Newton narrowing operators. Prace Naukowe Politechniki Warszawskiej. Elektronika 169, 111-119 (2009). KAEiOG 2009 Proceedings

24. Kubica, B.J.: Shared-memory parallelization of an interval equations systems solver - comparison of toos. Prace Naukowe Politechniki Warszawskiej. Elektronika 169, 121-128 (2009). KAEiOG, 2009 Proceedings

25. Kubica, B.J.: Interval methods for solving underdetermined nonlinear equations systems. Reliable Computing 15, 207-217 (2011). SCAN 2008 Proceedings

26. Kubica, B.J.: Exclusion regions in the interval solver of underdetemined nonlinear systems. Tech. Rep. 12-01, ICCE WUT (2012)

27. Kubica, B.J.: Tuning the multithreaded interval method for solving underdetermined systems of nonlinear equations. Lect. Notes Comput. Sci. 7204, 467-476 (2012). PPAM 2011 Proceedings

28. Kubica, B.J.: Excluding regions using Sobol sequences in an interval branch-and-prune method for nonlinear systems. Reliab. Comput. 19(4), 385-397 (2014). SCAN 2012 Proceedings

29. Kubica, B.J.: Using quadratic approximations in an interval method for solving underdetermined and well-determined nonlinear systems. Lect. Notes Comput. Sci. 8385, 623-633 (2014). PPAM 2013 Proceedings

30. Kubica, B.J., Woźniak, A.: Using the second-order information in Pareto-set computations of a multicriteria problem. Lect. Notes Comput. Sci. 7134, 137-147 (2012). PARA 2010 Proceedings

31. Meyn, K.H.: Solution of underdetermined nonlinear equations by stationary iteration methods. Numer. Math. 42, 161-172 (1983)

32. Neumaier, A.: The enclosure of solutions of parameter-dependent systems of equations. In: Reliability in Computing, pp. 269-286. Academic Press (1988)

33. Puget, J.F., Hentenryck, P.V.: A constraint satisfaction approach to a circuit design problem. J. Glob. Optim. 13(1), 75-93 (1998)

34. Ratschek, H., Rokne, J.: Experiments using interval analysis for solving a circuit design problem. J. Glob. Optim. 3(4), 501-518 (1993)

35. Rheinboldt, W.C.: Computation of critical boundaries on equilibrium manifolds. SIAM J. Numer. Anal. 19, 653-669 (1982)

36. Shary, S.P.: An interval linear tolerance problem. Autom. Remote. Control. 65, 1653-1666 (2004)

37. Shary, S.P.: Finite-dimensional Interval Analysis. XYZ. Electronic book (in Russian). (accessed 2014.05.15) (2013). URL http://www.nsc.ru/interval/Library/InteBooks/SharyBook.pdf 